\title{
ATLASGAL - Ammonia observations towards the southern Galactic plane ${ }^{\star}$
}

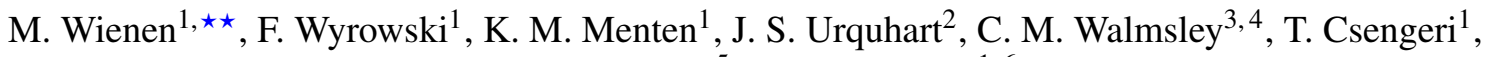 \\ B. S. Koribalski ${ }^{5}$, and F. Schuller ${ }^{1,6}$
}

\author{
1 Max-Planck-Institut für Radioastronomie, Auf dem Hügel 69, 53121 Bonn, Germany \\ e-mail: mwienen@mpifr-bonn.mpg.de \\ 2 School of Physical Sciences, University of Kent, Ingram Building, Canterbury, Kent CT2 7NH, UK \\ 3 Osservatorio Astrofisico di Arcetri, Largo E. Fermi, 5, 50125 Firenze, Italy \\ 4 Dublin Institute of Advanced Studies, Fitzwilliam Place 31, Dublin 2, Ireland \\ 5 Australia Telescope National Facility, CSIRO, P.O. Box 76, Epping, NSW 1710, Australia \\ 6 Alonso de Cordova 3107, Casilla 19001, Santiago 19, Chile
}

Received 22 April 2015 / Accepted 5 August 2017

\begin{abstract}
Context. The initial conditions of molecular clumps in which high-mass stars form are poorly understood. In particular, a more detailed study of the earliest evolutionary phases is needed. The APEX Telescope Large Area Survey of the whole inner Galactic disk at $870 \mu \mathrm{m}$, ATLASGAL, has therefore been conducted to discover high-mass star-forming regions at different evolutionary phases. Aims. We derive properties such as velocities, rotational temperatures, column densities, and abundances of a large sample of southern ATLASGAL clumps in the fourth quadrant.

Methods. Using the Parkes telescope, we observed the $\mathrm{NH}_{3}(1,1)$ to $(3,3)$ inversion transitions towards 354 dust clumps detected by ATLASGAL within a Galactic longitude range between $300^{\circ}$ and $359^{\circ}$ and a latitude within $\pm 1.5^{\circ}$. For a subsample of 289 sources, the $\mathrm{N}_{2} \mathrm{H}^{+}$(1-0) line was measured with the Mopra telescope.

Results. We measured a median $\mathrm{NH}_{3}(1,1)$ line width of $\sim 2 \mathrm{~km} \mathrm{~s}^{-1}$, rotational temperatures from 12 to $28 \mathrm{~K}$ with a mean of $18 \mathrm{~K}$, and source-averaged $\mathrm{NH}_{3}$ abundances from $1.6 \times 10^{-6}$ to $10^{-8}$. For a subsample with detected $\mathrm{NH}_{3}(2,2)$ hyperfine components, we found that the commonly used method to compute the $(2,2)$ optical depth from the $(1,1)$ optical depth and the $(2,2)$ to $(1,1)$ main beam brightness temperature ratio leads to an underestimation of the rotational temperature and column density. A larger median virial parameter of $\sim 1$ is determined using the broader $\mathrm{N}_{2} \mathrm{H}^{+}$line width than is estimated from the $\mathrm{NH}_{3}$ line width of $\sim 0.5$ with a general trend of a decreasing virial parameter with increasing gas mass. We obtain a rising $\mathrm{NH}_{3}(1,1) / \mathrm{N}_{2} \mathrm{H}^{+}$line-width ratio with increasing rotational temperature.

Conclusions. A comparison of $\mathrm{NH}_{3}$ line parameters of ATLASGAL clumps to cores in nearby molecular clouds reveals smaller velocity dispersions in low-mass than high-mass star-forming regions and a warmer surrounding of ATLASGAL clumps than the surrounding of low-mass cores. The $\mathrm{NH}_{3}(1,1)$ inversion transition of $49 \%$ of the sources shows hyperfine structure anomalies. The intensity ratio of the outer hyperfine structure lines with a median of $1.27 \pm 0.03$ and a standard deviation of 0.45 is significantly higher than 1, while the intensity ratios of the inner satellites with a median of $0.9 \pm 0.02$ and standard deviation of 0.3 and the sum of the inner and outer hyperfine components with a median of $1.06 \pm 0.02$ and standard deviation of 0.37 are closer to 1 .
\end{abstract}

Key words. submillimeter: ISM - surveys - ISM: molecules - ISM: kinematics and dynamics - stars: formation - stars: massive

\section{Introduction}

The earliest phases of high-mass star formation occur in the densest regions of giant molecular clouds. Several surveys (Burton et al. 1975; Cohen \& Thaddeus 1977; Bronfman et al. 1988) have established the large-scale distribution of molecular clouds in the Galaxy. Observations have also revealed the physical properties of these objects, such as their mass, which ranges from $10^{4}$ to $10^{6} M_{\odot}$, sizes of between 50 to $200 \mathrm{pc}$, and

\footnotetext{
$\star$ The final survey data (FITS files) and full Tables $1-3,5$ are available at the CDS via anonymous ftp to cdsarc.u-strasbg. fr (130.79.128.5) or via http://cdsarc.u-strasbg.fr/viz-bin/qcat?]/A+A/609/A125 $\star \star$ Member of the International Max Planck Research School (IMPRS) for Astronomy and Astrophysics at the Universities of Bonn and Cologne.
}

temperatures of $\sim 10 \mathrm{~K}$ (Dame et al. 2001). Clumps consisting of molecular gas that are embedded within these molecular clouds have high masses $\left(>500 M_{\odot}\right)$, high densities $\left(>10^{5} \mathrm{~cm}^{-3}\right)$, and low temperatures $(<20 \mathrm{~K}$; Bergin \& Tafalla 2007), and thus exhibit the initial conditions expected for high-mass star or cluster formation. While it is known that massive stars form in clusters, the detailed physical processes are little understood. In addition, because massive young stellar objects (YSOs) are rarer than low-mass stars according to the initial mass function (only $\sim 500$ are identified throughout the Galactic plane, Kroupa et al. 2013; Lumsden et al. 2013), they are more distant and heavily affected by extinction. Especially the earliest stages of high-mass star formation are not well known compared to an accepted evolutionary scenario explaining isolated low-mass star formation (André et al. 2000). 
While high-mass protostars evolve in massive dense cores within the clumps (Bontemps et al. 2010; Csengeri et al. 2011), their intense ultraviolet radiation heats, ionizes, and disrupts their natal molecular cloud. Embedded YSOs that have already formed an HII region have been studied in more detail because they can be observed in far-infrared and radio continuum surveys (Wood \& Churchwell 1989; Becker et al. 1990; Kurtz et al. 1994; Walsh et al. 1998; Hoare et al. 2012; Urquhart et al. 2013b). In addition to these regions that are in a more evolved phase of high-mass star formation, further progress required the observation of earlier stages. Surveys searched for the progenitors of ultracompact HII regions (UCHIIRs), thus very luminous $\left(>10^{3} L_{\odot}\right)$ infrared protostars, that are embedded in a high-mass envelope and harbour hot gas, but are not detected at centimetre wavelengths. Association of high-density gas around these sources reveals an envelope and the detection of a hot core, and water or methanol masers indicate hot gas. These high-mass protostellar objects have been analysed in several studies (Bronfman et al. 1996; Hunter et al. 2000; Brand et al. 2001; Sridharan et al. 2002; Lumsden et al. 2013; Urquhart et al. 2014b). Recently, massive dense cores and high-mass protostars at an early stage have been directly observed (Bontemps et al. 2010; Duarte-Cabral et al. 2013, 2014). Surveys for water and 6.7 GHz methanol maser emission detected numerous new high-mass star-forming regions (Plume et al. 1997; Szymczak et al. 2002; Sridharan et al. 2002; Caswell et al. 2011; Green et al. 2009). Other large-scale surveys conducted within the Galactic plane are the Spitzer GLIMPSE (Galactic Legacy Infrared Mid-Plane Survey Extraordinaire; Benjamin et al. 2003) between 3 to $8 \mu \mathrm{m}$ and the MIPSGAL survey (MIPS Galactic Plane Survey; Carey et al. 2009) at 24 and $70 \mu \mathrm{m}$. Longer wavelengths are observed by the BGPS (Bolocam Galactic Plane Survey; Aguirre et al. 2011) at $1.1 \mathrm{~mm}$ and Hi-GAL (Herschel Infrared Galactic Plane Survey; Molinari et al. 2010) from 70 to $500 \mu \mathrm{m}$.

Even younger stellar objects are still deeply embedded in an envelope and thus so cold that they cannot be detected at midinfrared wavelengths. They can be found in infrared dark clouds (IRDCs), which were discovered by two infrared satellites, ISO and MSX, as extinction features against the bright mid-infrared background (Egan et al. 1998; Perault et al. 1996). High column densities $\left(\sim 10^{23}-10^{25} \mathrm{~cm}^{-2}\right)$ and low temperatures $(<25 \mathrm{~K})$ of IRDCs (Carey et al. 1998, 2000; Pillai et al. 2006) indicate that they are in the earliest phases of star formation.

Surveys conducted toward massive star-forming regions have only traced one particular evolutionary phase, such as UCHIIRs or IRDCs (Wood \& Churchwell 1989; Vasyunina et al. 2009), or the targeted samples have been limited in number. To enlarge these samples and to trace the early, cold stages as well as later evolutionary phases, the first unbiased submillimeter-continuum survey of the whole inner Galactic disk, The APEX Telescope Large Area Survey of the Galaxy at $870 \mu \mathrm{m}$ (ATLASGAL; Schuller et al. 2009) was conducted. It observed the Galactic longitude range of $\pm 60^{\circ}$ and latitude of $\pm 1.5^{\circ}$ using the Large APEX Bolometer Camera (LABOCA; Siringo et al. 2007, 2008) with a beam width of 19.2" FWHM at the wavelength of $870 \mu \mathrm{m}$. ATLASGAL aims to derive physical properties of a statistically representative sample of objects at different evolutionary phases of high-mass star formation. To extract sources from the submillimeter (submm) maps, two algorithms are exploited: SExtractor (Bertin \& Arnouts 1996) provides global properties for clumps that are presented in the ATLASGAL Compact Source Catalogue (CSC; Contreras et al. 2013; Urquhart et al. 2014a), while the Gaussclumps algorithm
(Stutzki \& Guesten 1990; Kramer et al. 1998) separates compact emission from the more diffuse envelope (Csengeri et al. 2014). In addition to the identification of massive clumps, information from molecular line observations is also required. To derive the three-dimensional distribution of the dense material, for instance, distances and therefore velocities are necessary (Wienen et al. 2015). $\mathrm{NH}_{3}$ is a useful tool for this task because it is still present in the gas phase of the very dense $\left(\sim 10^{5} \mathrm{~cm}^{-3}\right.$; Beuther et al. 2002) and cold molecular cores (Tafalla et al. 2002), where other molecules such as CS and $\mathrm{CO}$ are partly frozen onto dust grains. In addition, $\mathrm{NH}_{3}$ is an important probe to measure the temperature of molecular clouds because they exhibit low temperatures $(\sim 20 \mathrm{~K})$, at which the $\mathrm{NH}_{3}$ inversion transitions in the lowest metastable $(J, K)$ rotational energy levels are excited (Ho \& Townes 1983). The splitting of the inversion transitions into different hyperfine structure components allows deriving the optical depth and column density. The rotational temperature of the gas within molecular clumps can then be determined from the line ratios between different inversion transitions and the optical depth. The line width can also be used to determine the stability of the clumps from an analysis of their virial masses.

Previous studies have used ammonia to analyse the physical properties of nearby molecular clouds (Rosolowsky et al. 2008; Friesen et al. 2009; Johnstone et al. 2010). Various observations of ammonia have also been conducted toward high-mass star-forming regions (Mangum et al. 1992; Wilson et al. 1993; Cesaroni et al. 1994; Fontani et al. 2004; Ragan et al. 2011). These studies tend to focus on a single stage of high-mass star formation such as IRDCs, YSOs, or HII regions (Peretto \& Fuller 2009; Chira et al. 2013; Moscadelli \& Goddi 2014; Zhang et al. 2014), contain a small number of sources (Pillai et al. 2006; Wu et al. 2010b; Giannetti et al. 2013; Lu et al. 2014), or are confined to the first quadrant (Dunham et al. 2011; Urquhart et al. 2011, 2015). A shallow low-resolution $\left(2^{\prime}\right)$ survey of $\mathrm{NH}_{3}(1,1)$ and $(2,2)$ has been part of the $\mathrm{H}_{2} \mathrm{O}$ southern Galactic Plane Survey (HOPS; Walsh et al. 2011; Purcell et al. 2012) in the fourth quadrant. These measurements cover the Galactic longitude range of the ATLASGAL survey, but are confined to a Galactic latitude of $\pm 0.5^{\circ}$.

We observed ammonia towards a large sample of ATLASGAL sources in different evolutionary phases of high-mass star formation in the fourth quadrant, which allows a statistical analysis of derived line parameters. First, the $\mathrm{NH}_{3}(1,1)$ to $(3,3)$ line observations were made towards 862 northern ATLASGAL clumps in the first quadrant, within $|b| \leq 1.5^{\circ}$ and $l=5^{\circ}-60^{\circ}$; the results are presented in Wienen et al. (2012). We extended this survey to the fourth quadrant and observed 354 southern ATLASGAL sources within $|b| \leq 1.5^{\circ}$ and $l=300^{\circ}-359^{\circ}$. This article focuses on physical properties that could not be studied for the northern sources. The structure of the paper is as follows.

Section 2 presents the $\mathrm{NH}_{3}$ observations and data reduction. We analyse properties derived from the $\mathrm{NH}_{3}$ inversion lines such as the velocity, line width, rotational temperature, and column density and show their variation with galactocentric radius in Sect. 3. We investigate the dependence of these line parameters on the environment within molecular clouds at different distances in Sect. 4. In addition, we compare virial parameters derived from the $\mathrm{NH}_{3}$ line width with those calculated from the line width of a higher density tracer, $\mathrm{N}_{2} \mathrm{H}^{+}$. Furthermore, we analyse anomalies in the relative hyperfine satellite intensities of the $\mathrm{NH}_{3}$ $(1,1)$ inversion transition. Section 5 presents a summary of our work. 
Table 1. $\mathrm{NH}_{3}(1,1)$ line parameters.

\begin{tabular}{lllllll}
\hline \hline & $\begin{array}{l}\mathrm{RA}^{1} \\
\text { Name }\end{array}$ & $\begin{array}{l}\mathrm{Dec}^{1} \\
(\mathrm{~J} 2000)\end{array}$ & $\tau(1,1)$ & $\begin{array}{l}v(1,1) \\
\left(\mathrm{km} \mathrm{s}^{-1}\right)\end{array}$ & $\begin{array}{l}\Delta v(1,1) \\
\left(\mathrm{km} \mathrm{s}^{-1}\right)\end{array}$ & $\begin{array}{l}T_{\mathrm{MB}}(1,1) \\
(\mathrm{K})\end{array}$ \\
\hline G300.72+1.20 & 123250.23 & -613527.4 & $1.98( \pm 0.89)$ & $-43.96( \pm 0.19)$ & $2.89( \pm 0.38)$ & $0.18( \pm 0.05)$ \\
G300.82+1.15 & 123340.83 & -613852.9 & $2.06( \pm 0.39)$ & $-42.72( \pm 0.05)$ & $1.78( \pm 0.11)$ & $0.41( \pm 0.04)$ \\
G300.91+0.88 & 123414.23 & -615522.7 & $1.37( \pm 0.23)$ & $-40.87( \pm 0.04)$ & $2.52( \pm 0.09)$ & $0.82( \pm 0.06)$ \\
G300.97+1.15 & 123452.75 & -613947.9 & $0.71( \pm 0.37)$ & $-42.51( \pm 0.07)$ & $2.16( \pm 0.16)$ & $0.28( \pm 0.03)$ \\
G301.01+1.11 & 123514.97 & -614155.4 & $1.36( \pm 0.37)$ & $-42.02( \pm 0.06)$ & $2.22( \pm 0.13)$ & $0.56( \pm 0.06)$ \\
G301.14-0.23 & 123534.87 & -630231.1 & $0.10( \pm 0.12)$ & $-39.58( \pm 0.16)$ & $3.42( \pm 0.31)$ & $0.14( \pm 0.03)$ \\
G301.12+0.96 & 123601.82 & -615127.8 & $1.41( \pm 0.19)$ & $-40.13( \pm 0.02)$ & $1.56( \pm 0.05)$ & $1.10( \pm 0.05)$ \\
G301.12+0.98 & 123602.85 & -615026.0 & $1.68( \pm 0.14)$ & $-40.14( \pm 0.01)$ & $1.33( \pm 0.03)$ & $1.49( \pm 0.05)$ \\
G301.14+1.01 & 123614.07 & -614839.3 & $1.72( \pm 0.17)$ & $-39.89( \pm 0.03)$ & $2.33( \pm 0.05)$ & $0.95( \pm 0.05)$ \\
G301.68+0.25 & 124032.82 & -623554.8 & $3.08( \pm 0.45)$ & $-37.67( \pm 0.04)$ & $1.38( \pm 0.07)$ & $0.64( \pm 0.05)$ \\
G301.74+1.10 & 124119.90 & -614441.1 & $1.72( \pm 0.14)$ & $-39.49( \pm 0.02)$ & $1.90( \pm 0.04)$ & $0.47( \pm 0.02)$ \\
G301.81+0.78 & 124153.36 & -620412.3 & $0.42( \pm 0.63)$ & $-37.13( \pm 0.08)$ & $1.57( \pm 0.21)$ & $0.28( \pm 0.04)$ \\
G302.39+0.28 & 124644.26 & -623511.9 & $1.34( \pm 0.28)$ & $-43.19( \pm 0.04)$ & $1.87( \pm 0.09)$ & $0.44( \pm 0.03)$ \\
G304.20+1.34 & 130206.13 & -613025.3 & $1.54( \pm 0.51)$ & $-43.63( \pm 0.05)$ & $1.34( \pm 0.12)$ & $0.42( \pm 0.05)$ \\
G304.76+1.34 & 130645.70 & -612839.9 & $0.67( \pm 0.21)$ & $-26.80( \pm 0.02)$ & $1.43( \pm 0.06)$ & $0.90( \pm 0.04)$ \\
\hline
\end{tabular}

Notes. Errors are given in parentheses. The full table is available at the CDS. ${ }^{(1)}$ Units of right ascension are hours, minutes, and seconds, and units of declination are degrees, arcminutes, and arcseconds.

\section{Observations and data reduction}

\subsection{Parkes observations}

A flux-limited subsample was selected from a preliminary ATLASGAL point-source catalogue similar to the observations made with the Effelsberg telescope described in Wienen et al. (2012). We selected clumps with peak fluxes above a threshold of $1.2 \mathrm{Jy} /$ beam in the fourth quadrant, while our northern $\mathrm{NH}_{3}$ sample exhibits peak flux densities above $0.4 \mathrm{Jy} /$ beam. In 2009, ammonia observations of a total of 354 dust clumps located in the Galactic longitude range between $300^{\circ}$ and $359^{\circ}$ and latitude within $\pm 1.5^{\circ}$ were conducted.

We observed the $\mathrm{NH}_{3}(1,1),(2,2)$, and $(3,3)$ inversion transitions using the Parkes $64 \mathrm{~m}$ telescope from 20 to 27 June, 2009. The frontend was a $13 \mathrm{~mm}$ receiver that covers the frequency range from 16 to $26 \mathrm{GHz}$. The beam width (FWHM) at the $\mathrm{NH}_{3}$ $(1,1)$ to $(3,3)$ line frequencies at $\sim 24 \mathrm{GHz}$ is $61^{\prime \prime}$. The spectrometer was a Digital Filter Bank (DFB3) with a bandwidth of $256 \mathrm{MHz}$, which results in a spectral resolution of $\sim 0.4 \mathrm{~km} \mathrm{~s}^{-1}$. Two polarizations of each of the three $\mathrm{NH}_{3}$ transitions were measured simultaneously. The observations were conducted in position-switching mode with a constant offset of $\sim 15^{\prime}$ with a total integration time of about $8 \mathrm{~min}$ for each source, pointing and focus were measured approximately every hour. To calibrate the data, a reference source, G15.66-0.50, was measured in each observing session. To connect the calibration to the data observed with Effelsberg (Wienen et al. 2012), we compared the northern and southern $(3,3)$ line intensities of G15.66-0.50, which is assumed to be compact. This gives a calibration factor of 3.47 that also accounts for the atmospheric opacity, which is multiplied to the data. These were then corrected for the gain-elevation curve. The variation in main-beam brightness temperatures between the different observing days gives a calibration error of $\sim 7 \%$. Typical system temperatures were about $50 \mathrm{~K}$. We measured an rms noise level between 10 and $130 \mathrm{mK}$ at a velocity resolution of $0.4 \mathrm{~km} \mathrm{~s}^{-1}$.

The $\mathrm{NH}_{3}$ spectra were read into the CLASS software ${ }^{1}$, which we used to reduce the spectra. The ammonia lines of each source

\footnotetext{
Available at http://www . iram. fr/IRAMFR/GILDAS
}

can be observed in two polarizations, which are averaged together. Since position-switching was performed as observing mode, the baseline is more stable than it was for the northern $\mathrm{NH}_{3}$ measurements, for which we used frequency-switching (Wienen et al. 2012). As there are still some fluctuations in the baseline, they were corrected by subtracting a polynomial baseline of order 3 to 7 over a velocity range of $100 \mathrm{~km} \mathrm{~s}^{-1}$ for the $\mathrm{NH}_{3}(1,1)$ line and over $70 \mathrm{~km} \mathrm{~s}^{-1}$ for the $\mathrm{NH}_{3}(2,2)$ line. We used the method described by Wienen et al. (2012) to place windows around the hyperfine structure components for the northern $\mathrm{NH}_{3}$ lines. We followed the procedure described in Wienen et al. (2012) to derive line parameters with fits in CLASS of the ammonia $(1,1)$ lines that include 18 hyperfine structure components. In contrast to the northern sources, a small southern subsample is detected in the $(2,2)$ hyperfine structure. We did not use a single-Gaussian fitting to the main line of the $(2,2)$ transition as for the northern sources, but fitted all the 22 hyperfine structure components of the whole southern sample (see Sects. 3.3 and 3.4 for details). The optical depth of the $(3,3)$ line could not be derived because we did not detect the hyperfine structure, and we fitted a single Gaussian to the main line of the $(3,3)$ transition. The derived positions of the sources, the optical depths of the $\mathrm{NH}_{3}(1,1)$ lines, $\tau(1,1)$, their LSR velocities, $v(1,1)$, FWHM line widths, $\Delta v(1,1)$, and main-beam brightness temperatures, $T_{\mathrm{MB}}(1,1)$, with their formal fit errors are given in Table 1 . The LSR velocities, FWHM line widths, and main-beam brightness temperatures of the $(2,2)$ and $(3,3)$ lines together with their formal fit errors can be found in Table 2. Using the standard formulation for $\mathrm{NH}_{3}$ spectra (Ho \& Townes 1983; Ungerechts et al. 1986), we determined physical parameters such as the rotational temperature $\left(T_{\text {rot }}\right)$, kinetic temperature $\left(T_{\text {kin }}\right)$, and ammonia column density $\left(N_{\mathrm{NH}_{3}}\right)$, see Sect. 3 . Table 3 contains the parameters with the errors $(1 \sigma)$ calculated from Gaussian error propagation.

\subsection{Mopra observations}

We selected a flux-limited subsample of clumps from a preliminary ATLASGAL compact source catalogue. It ensured high 
Table 2. $\mathrm{NH}_{3}(2,2)$ and $(3,3)$ line parameters.

\begin{tabular}{lllllll}
\hline \hline & $\begin{array}{l}v(2,2) \\
\left(\mathrm{km} \mathrm{s}^{-1}\right)\end{array}$ & $\begin{array}{l}\Delta v(2,2) \\
\left(\mathrm{km} \mathrm{s}^{-1}\right)\end{array}$ & $\begin{array}{l}T_{\mathrm{MB}}(2,2) \\
(\mathrm{K})\end{array}$ & $\begin{array}{l}v(3,3) \\
\left(\mathrm{km} \mathrm{s}^{-1}\right)\end{array}$ & $\begin{array}{l}\Delta v(3,3) \\
\left(\mathrm{km} \mathrm{s}^{-1}\right)\end{array}$ & $\begin{array}{l}T_{\mathrm{MB}}(3,3) \\
(\mathrm{K})\end{array}$ \\
\hline G300.72+1.20 & - & - & $<0.05$ & - & - & $<0.05$ \\
G300.82+1.15 & $-42.54( \pm 0.12)$ & $1.83( \pm 0.28)$ & $0.18( \pm 0.03)$ & - & - & $<0.04$ \\
G300.91+0.88 & $-41.20( \pm 0.14)$ & $2.77( \pm 0.35)$ & $0.33( \pm 0.06)$ & - & - & $<0.07$ \\
G300.97+1.15 & $-42.57( \pm 0.13)$ & $2.49( \pm 0.24)$ & $0.19( \pm 0.03)$ & $-42.76( \pm 0.18)$ & $4.09( \pm 0.45)$ & $0.14( \pm 0.03)$ \\
G301.01+1.11 & $-41.84( \pm 0.19)$ & $3.40( \pm 0.41)$ & $0.18( \pm 0.04)$ & $-41.44( \pm 0.23)$ & $2.61( \pm 0.57)$ & $0.12( \pm 0.04)$ \\
G301.14-0.23 & $-38.49( \pm 0.44)$ & $5.38( \pm 1.42)$ & $0.07( \pm 0.03)$ & - & - & $<0.03$ \\
G301.12+0.96 & $-40.28( \pm 0.06)$ & $1.77( \pm 0.14)$ & $0.48( \pm 0.05)$ & $-40.81( \pm 0.14)$ & $1.43( \pm 0.34)$ & $0.16( \pm 0.04)$ \\
G301.12+0.98 & $-40.19( \pm 0.04)$ & $1.39( \pm 0.09)$ & $0.66( \pm 0.05)$ & $-40.29( \pm 0.43)$ & $3.44( \pm 1.31)$ & $0.10( \pm 0.05)$ \\
G301.14+1.01 & $-40.02( \pm 0.09)$ & $2.65( \pm 0.17)$ & $0.38( \pm 0.05)$ & $-40.21( \pm 0.14)$ & $2.86( \pm 0.41)$ & $0.27( \pm 0.05)$ \\
G301.68+0.25 & $-37.64( \pm 0.14)$ & $1.70( \pm 0.34)$ & $0.22( \pm 0.05)$ & - & - & $<0.05$ \\
G301.74+1.10 & $-39.57( \pm 0.07)$ & $2.18( \pm 0.13)$ & $0.17( \pm 0.02)$ & $-39.72( \pm 0.17)$ & $1.66( \pm 0.45)$ & $0.06( \pm 0.02)$ \\
G301.81+0.78 & $-37.79( \pm 0.16)$ & $0.95( \pm 0.26)$ & $0.14( \pm 0.05)$ & - & - & $<0.05$ \\
G302.39+0.28 & $-43.20( \pm 0.10)$ & $2.15( \pm 0.24)$ & $0.19( \pm 0.03)$ & - & - & $<0.03$ \\
G304.20+1.34 & $-43.71( \pm 0.21)$ & $2.21( \pm 0.50)$ & $0.16( \pm 0.05)$ & - & - & $<0.04$ \\
G304.76+1.34 & $-26.37( \pm 0.11)$ & $1.37( \pm 0.26)$ & $0.21( \pm 0.05)$ & - & - & $<0.06$ \\
\hline
\end{tabular}

Notes. Errors are given in parentheses. The full table is available at the CDS.

Table 3. Parameters derived from the $\mathrm{NH}_{3}(1,1)$ to $(3,3)$ inversion transitions.

\begin{tabular}{llll}
\hline \hline & $\begin{array}{l}T_{\text {rot }} \\
\text { Name }\end{array}$ & $\begin{array}{l}T_{\text {kin }} \\
(\mathrm{K})\end{array}$ & $\begin{array}{l}\log _{10}\left(N_{\mathrm{NH}_{3}}\right) \\
\left(\mathrm{cm}^{-2}\right)\end{array}$ \\
\hline G300.72+1.20 & - & - & - \\
G300.82+1.15 & $15.3( \pm 1.7)$ & $17.3( \pm 2.4)$ & $15.3( \pm 0.09)$ \\
G300.91+0.88 & $15.8( \pm 1.5)$ & $18.1( \pm 2.2)$ & $15.28( \pm 0.08)$ \\
G300.97+1.15 & $22.8( \pm 3.5)$ & $29.9( \pm 6.8)$ & $15.03( \pm 0.24)$ \\
G301.01+1.11 & $14.4( \pm 1.7)$ & $16.1( \pm 2.3)$ & $15.21( \pm 0.12)$ \\
G301.14-0.23 & $21.3( \pm 5.6)$ & $27.0( \pm 10.3)$ & $14.35( \pm 0.54)$ \\
G301.12+0.96 & $16.5( \pm 1.0)$ & $19.1( \pm 1.5)$ & $15.09( \pm 0.06)$ \\
G301.12+0.98 & $16.0( \pm 0.7)$ & $18.4( \pm 1.0)$ & $15.09( \pm 0.04)$ \\
G301.14+1.01 & $15.2( \pm 1.0)$ & $17.2( \pm 1.4)$ & $15.34( \pm 0.04)$ \\
G301.68+0.25 & $12.5( \pm 1.2)$ & $13.6( \pm 1.6)$ & $15.36( \pm 0.07)$ \\
G301.74+1.10 & $14.4( \pm 0.7)$ & $16.0( \pm 1.0)$ & $15.25( \pm 0.04)$ \\
G301.81+0.78 & $20.6( \pm 4.9)$ & $25.8( \pm 8.7)$ & $14.62( \pm 0.66)$ \\
G302.39+0.28 & $16.4( \pm 1.5)$ & $19.0( \pm 2.2)$ & $15.15( \pm 0.09)$ \\
G304.20+1.34 & $15.2( \pm 2.3)$ & $17.3( \pm 3.2)$ & $15.05( \pm 0.15)$ \\
G304.76+1.34 & $14.0( \pm 1.2)$ & $15.5( \pm 1.6)$ & $14.72( \pm 0.14)$ \\
G305.10+0.09 & $22.5( \pm 5.8)$ & $29.3( \pm 11.2)$ & $14.99( \pm 0.47)$ \\
\hline
\end{tabular}

Notes. Errors are given in parentheses. The full table is available at the CDS.

enough column densities for the Mopra line detections and a coverage of all phases of high-mass star formation. The sample consists of sources with an MSX infrared association within a distance of $30^{\prime \prime}$ and peak fluxes above a limit of $1.75 \mathrm{Jy} / \mathrm{beam}$, and cold clumps without MSX association and peak fluxes above $1.2 \mathrm{Jy} / \mathrm{beam}$. We observed a total of 700 ATLASGAL clumps within $l=300^{\circ}-359^{\circ}$ and $|b| \leq 1.5^{\circ}$ in 2009 and 2010 .

The $\mathrm{N}_{2} \mathrm{H}^{+}(1-0)$ line was observed using the Mopra $22 \mathrm{~m}$ Radiotelescope ${ }^{2}$. The frontend was a $3 \mathrm{~mm}$ HEMT receiver with a frequency range of between 76 and $117 \mathrm{GHz}$. The UNSW Mopra spectrometer (MOPS) consists of four $2.2 \mathrm{GHz}$ bands that overlap slightly and result in a total of $\sim 8 \mathrm{GHz}$ continuous bandwidth. We centred the $3 \mathrm{~mm}$ band on $89.3 \mathrm{GHz}$, covering the frequency range from $\sim 85.2 \mathrm{GHz}$ to $\sim 93.4 \mathrm{GHz}$. The FWHM at

\footnotetext{
2 Mopra is part of the Australia Telescope National Facility.
}

the frequency of the $\mathrm{N}_{2} \mathrm{H}^{+}(1-0)$ line at $\sim 90 \mathrm{GHz}$ is $38^{\prime \prime}$. MOPS was used in the broadband mode with a velocity resolution of $0.9 \mathrm{~km} \mathrm{~s}^{-1}$ of each $2.2 \mathrm{GHz}$ band.

MOPS was able to measure two polarizations of the $\mathrm{N}_{2} \mathrm{H}^{+}$ line simultaneously. We conducted pointed observations in position-switching mode. Using ATLASGAL and MSX maps, we searched around each source for an offset position that was free of emission, and used a constant offset of $\pm 5^{\circ}$ in longitude or latitude. Each source was observed with a total integration time of $\sim 15 \mathrm{~min}$, which gives an rms noise level of $24 \mathrm{mK}$ on average at a velocity resolution of $0.9 \mathrm{~km} \mathrm{~s}^{-1}$. Line pointings on $\mathrm{SiO}$ masers were measured every hour, a reference spectrum of G327 and M17 was taken each day.

The AsAP package ${ }^{3}$ was used for an initial processing of the data. This included processing of the on-off observing mode, the time and polarization averaging, and baseline subtraction. The data were calibrated to the $T_{\mathrm{A}}^{*}$ temperature scale that is transformed into $T_{\mathrm{MB}}$ by correcting for the beam efficiency of 0.49 (Ladd et al. 2005). For the subsequent analysis the data were exported to the CLASS software from the GILDAS package.

\section{Results for and analysis of the ammonia sample in the fourth quadrant}

\subsection{Ammonia line parameters}

Of the 354 clumps observed, 315 were detected in $\mathrm{NH}_{3}(1,1)$ (89\%), 262 sources $(74 \%)$ in $\mathrm{NH}_{3}(2,2)$, and 187 clumps $(53 \%)$ in the $(3,3)$ line with a signal-to-noise ratio $(\mathrm{S} / \mathrm{N})>3$. Most $\mathrm{NH}_{3}$ $(1,1)$ lines clearly show hyperfine structure, although it can be detected only in a few $(2,2)$ lines and is too weak to be observed in the $(3,3)$ lines. Examples of some ammonia spectra are displayed in Fig. 1. A few sources, such as G351.45+0.66, G326.64+0.61, and G327.29-0.58 in the upper panel, exhibit strong $\mathrm{NH}_{3}$ lines, about $10 \%$ have an $\mathrm{S} / \mathrm{N}$ of the $(1,1)$ line greater than 30 . On the other hand, Fig. 1 also shows example spectra with their fit for clumps with weak $(3,3)$ lines, which are G343.50+0.02, G345.26-0.03, and G348.76-0.95 in the lower

\footnotetext{
3 http://www.atnf.csiro.au/news/newsletter/jun06/ ASAP.htm
} 

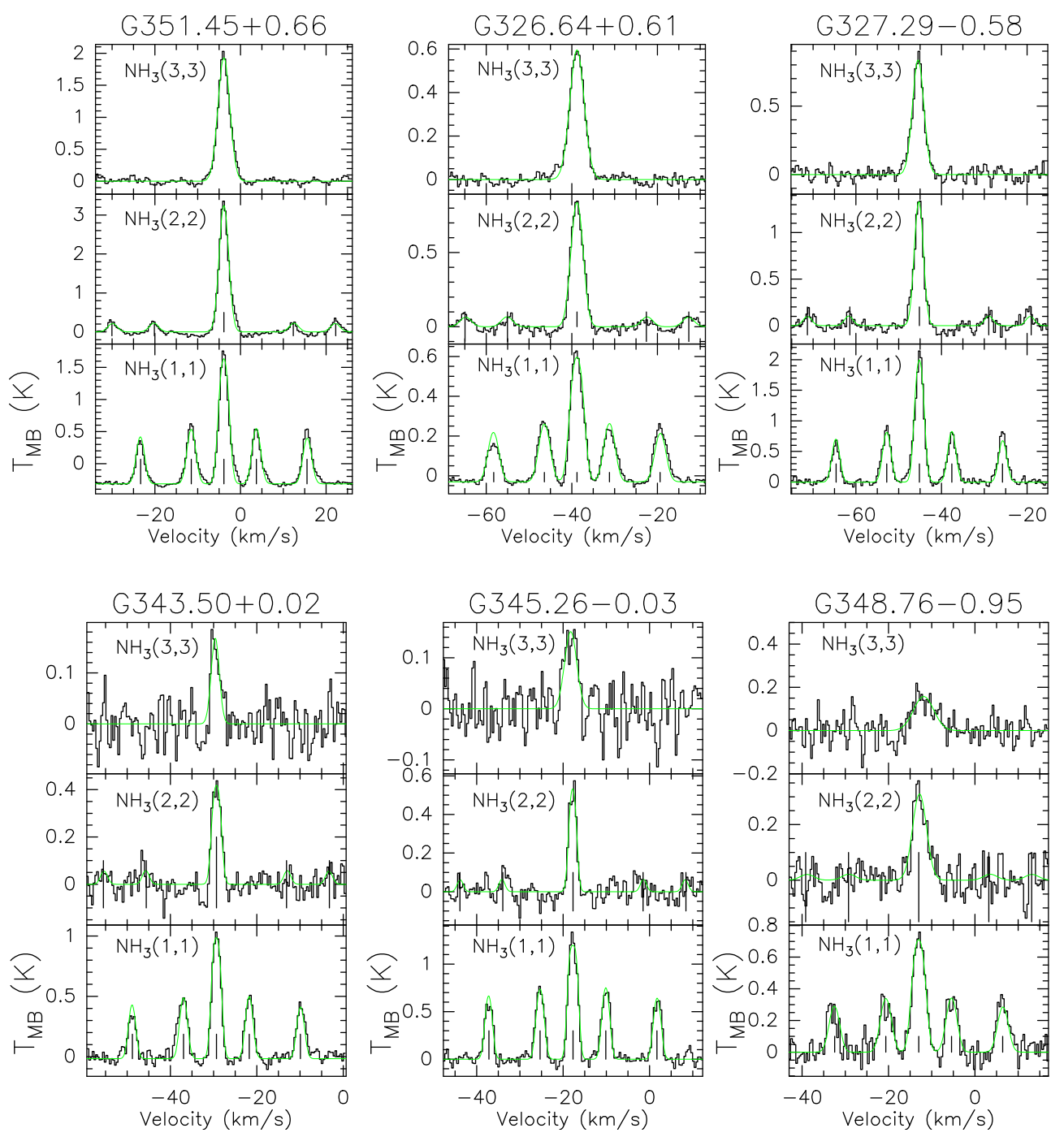

Fig. 1. Reduced and calibrated spectra of observed $\mathrm{NH}_{3}(1,1),(2,2)$, and $(3,3)$ inversion transitions; the fit is shown in green. The frequencies of the $(1,1)$ and $(2,2)$ main line and satellites are indicated by straight lines.

panel, their $\mathrm{S} / \mathrm{Ns}$ of the $(3,3)$ line are $\sim 4$. A comparison of the peak intensities of the different lines given in Tables 1 and 2 shows that the average main-beam brightness temperature of the $(2,2)$ line is $67 \%$ of the average $(1,1)$ peak intensity, while the average $(3,3)$ main-beam brightness temperature is $40 \%$ of the average $(1,1)$ peak intensity. This reveals that we are probing a range of excitation conditions and thus of star formation activity, which indicates that our sample consists of clumps in various evolutionary phases of high-mass star formation and in different environments.

In addition, we detect two different velocity components in nine $\mathrm{NH}_{3}$ spectra, that is, in $\mathrm{G} 328.2-0.55, \mathrm{G} 336.8+0.03$, and $\mathrm{G} 350.1+0.09$. These result from different clouds located on the same line of sight at different distances. We fit a model including two velocity components to them and denote them with "a" and "b" after the source name in Tables 1 to 3 .

\subsection{Ammonia velocities}

We illustrate the $\mathrm{NH}_{3}$ velocity and Galactic longitude range of the sources observed in the first quadrant (Wienen et al. 2012) and in the fourth quadrant in Fig. 2. The plot shows that more observations were conducted toward the northern than the southern ATLASGAL clumps. Most southern $\mathrm{NH}_{3}$ velocities range from
$5 \mathrm{~km} \mathrm{~s}^{-1}$ to $-120 \mathrm{~km} \mathrm{~s}^{-1}$ with a smaller number of extreme velocities than in the north. Only two clumps, G354.72+0.30 and $\mathrm{G} 354.75+0.37$, have high velocities of about $99 \mathrm{~km} \mathrm{~s}^{-1}$. These are located close to Bania's Clump 1 (Bania 1977) and are indicated as white points in Fig. 2; it is suggested that they are likely in the Galactic bar because of their high velocities (Caswell et al. 2010). The background in Fig. 2 shows CO (1-0) emission reported by Dame et al. (2001) and indicates a good correlation of most clumps with the $\mathrm{CO}$ intensity, which traces giant molecular clouds. The pink straight line denotes the $5 \mathrm{kpc}$ molecular ring that is the most massive and active star-forming molecular gas accumulation in our Galaxy (Simon et al. 2006). The $\mathrm{NH}_{3}$ radial velocities are combined with the Galactic rotation model (Brand \& Blitz 1993) to determine kinematic distances. We distinguish between near and far distances by examining HI selfabsorption and HI absorption towards a large sample of ATLASGAL sources, which gives an unbiased three-dimensional view of high-mass star-forming regions in the first and fourth quadrant (Wienen et al. 2015).

\subsection{Line width}

The $\mathrm{NH}_{3}(1,1)$ FWHM line widths lie between $0.8 \mathrm{~km} \mathrm{~s}^{-1}$ and $\sim 5 \mathrm{~km} \mathrm{~s}^{-1}$, while the $(2,2)$ inversion transitions have widths of 


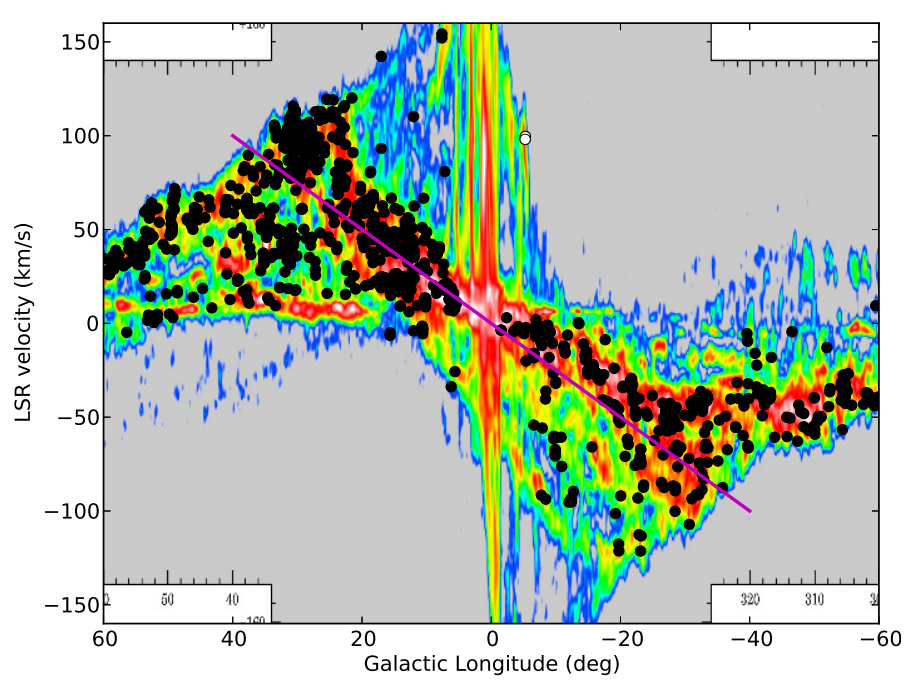

Fig. 2. Galactic longitude and LSR velocities of detected northern and southern sources are shown with CO (1-0) emission (Dame et al. 2001) in the background. The pink straight line indicates the $5 \mathrm{kpc}$ molecular ring (Simon et al. 2006). Two sources associated with Bania's Clump 1 are illustrated as white points.

up to $8 \mathrm{~km} \mathrm{~s}^{-1}$. Although the backend spectral resolution of the southern observations of $0.4 \mathrm{~km} \mathrm{~s}^{-1}$ is better than that of the northern $\mathrm{NH}_{3}$ sample of $0.7 \mathrm{~km} \mathrm{~s}^{-1}$, the narrowest $(1,1) \mathrm{FWHM}$ line widths of the southern and northern clumps are similar, $\sim 0.8 \mathrm{~km} \mathrm{~s}^{-1}$. The average of measured southern $(1,1)$ line widths is $\sim 2 \mathrm{~km} \mathrm{~s}^{-1}$, which agrees with the average obtained in the first quadrant and has mainly non-thermal contributions. The range of the $(2,2)$ and $(3,3)$ line widths is similar, the $(2,2)$ lines exhibit widths from $0.7 \mathrm{~km} \mathrm{~s}^{-1}$ to $8 \mathrm{~km} \mathrm{~s}^{-1}$ and the $(3,3)$ lines from $0.8 \mathrm{~km} \mathrm{~s}^{-1}$ to $7.6 \mathrm{~km} \mathrm{~s}^{-1}$. However, a comparison shows that most ATLASGAL sources have a larger width of the $(3,3)$ inversion transition than of the $(2,2)$ line. Clumps emitting a $\mathrm{NH}_{3}$ $(3,3)$ line must be warmer as a high temperature is needed to excite the $(3,3)$ inversion transition. This indicates that star formation activity heats these sources in the innermost parts of the clump where the density and turbulence are highest.

The $\mathrm{NH}_{3}(2,2)$ hyperfine components are clearly detected towards 27 sources in the fourth quadrant. We used a hyperfine structure fit for this subsample, which allowed us to measure the $(2,2)$ optical depth. We give the optical depth, $\tau(2,2)$, and the line width obtained from the hyperfine structure fit of the $\mathrm{NH}_{3}$ $(2,2)$ line, $\Delta v_{\mathrm{hfs}}(2,2)$, for the subsample of 31 sources in Table 4.

The $(1,1)$ line widths are plotted against the $(2,2)$ line widths for the subsample with detected hyperfine structure of the $(2,2)$ line in Fig. 3. For most sources, the two line widths agree, while a few clumps exhibit broader $(2,2)$ than $(1,1)$ line widths. Some of them with the largest line broadening show signs of star formation activity, but a few are not detected at $24 \mu \mathrm{m}$. Among these " $24 \mu \mathrm{m}$ dark" clumps are G326.64+0.61, for which Peretto \& Fuller (2009) did not find any hint of star formation activity within this IRDC, and G354.62+0.47, which is associated with $6.7 \mathrm{GHz}$ methanol maser emission (Urquhart et al. 2013a). Among the infrared bright sources are G333.02+0.77, which is located within an IRDC and associated with a $24 \mu \mathrm{m}$ source (Peretto \& Fuller 2009), G351.45+0.66, a bright hot core within the NGC 6334 molecular cloud complex (Rodriguez et al. 1982), and G327.29-0.58, a bright hot core within a high-mass star-forming complex (Minier et al. 2009; Lee et al. 2012).

Guided by the method described by Rosolowsky et al. (2008), we calculated the $(2,2)$ optical depth, $\tau(2,2)$, from the

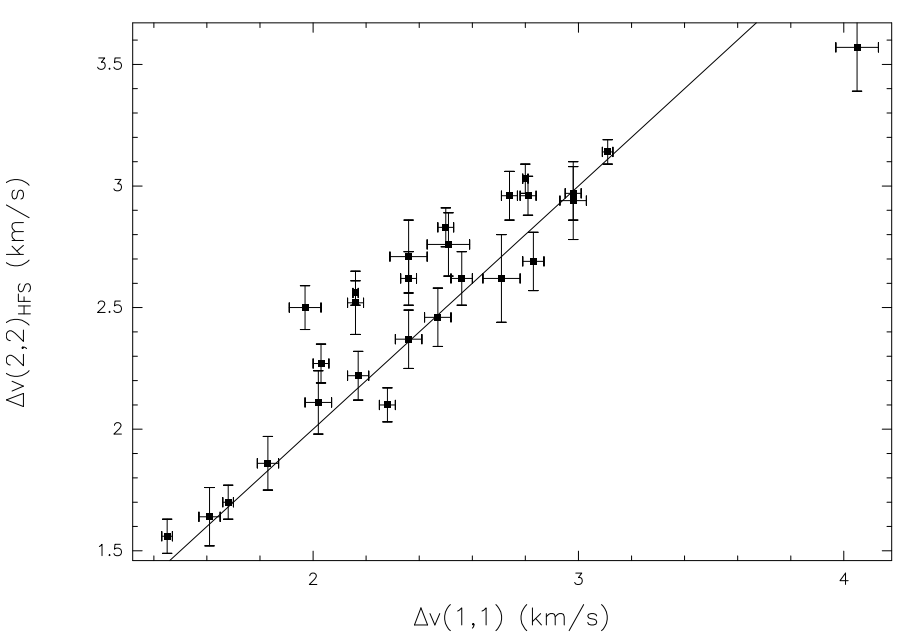

Fig. 3. Correlation plot of $(1,1)$ and $(2,2)$ line widths for sources for which the hyperfine structure of the $\mathrm{NH}_{3}(2,2)$ line is detected. The solid line presents equal line widths.

$(1,1)$ and $(2,2)$ main-beam brightness temperatures, $T_{\mathrm{MB}}$, and the $(1,1)$ optical depth, $\tau(1,1)$ :

$\tau(2,2)=\ln \left(1-\frac{T_{\mathrm{MB}}(2,2)}{T_{\mathrm{MB}}(1,1)}(1-\exp (-\tau(1,1)))\right)$.

This was used as a fixed parameter in the hyperfine structure fit of the $(2,2)$ transition of the whole ATLASGAL sample in the fourth quadrant. The derived $(2,2)$ line widths are given in Table 2 . The intrinsic $(2,2)$ line widths resulting from a hyperfine structure fit with the measured $(2,2)$ optical depth against those derived from the hyperfine structure fit with fixed $(2,2)$ optical depth are compared in Fig. 4. It reveals a constant offset from equal line widths, explained in Sect. 3.4, with broader $(2,2)$ line widths resulting from the hypefine structure fit with fixed $(2,2)$ optical depth.

\subsection{Rotational temperature and source-averaged $\mathrm{NH}_{3}$ column density}

Assuming equal beam-filling factors, we calculated the rotational temperature between the $(1,1)$ and $(2,2)$ inversion transition using (Ho \& Townes 1983)

$T_{\text {rot }}=\frac{-41.5}{\ln \left(-0.282 \frac{\tau(2,2)}{\tau(1,1)}\right)}$,

where $\tau(1,1)$ and $\tau(2,2)$ are the optical depths of the $(1,1)$ and $(2,2)$ main lines. Because we did not detect the hyperfine structure of most $(2,2)$ lines, we computed the $(2,2)$ optical depth from Eq. (1). The same excitation temperature was assumed for the $(1,1)$ and $(2,2)$ lines, $T_{\mathrm{ex}}$, in Eqs. (1) and (2). We tested this assumption for the subsample given in Sect. 3.3, for which the optical depth could be derived from the detected $(1,1)$ and $(2,2)$ hyperfine components. The excitation temperature can be computed from

$T_{\mathrm{ex}}=\frac{T_{\mathrm{MB}}(1,1)}{1-\exp (-\tau(1,1))}+T_{\mathrm{bg}}$

with $T_{\mathrm{bg}}=2.73 \mathrm{~K}$ and assuming a beam-filling factor of 1 . The excitation temperature of the $\mathrm{NH}_{3}(2,2)$ line is on average lower but still within $10-20 \%$ of the $(1,1)$ excitation temperature value. It is unclear whether this small difference is due to an actual 


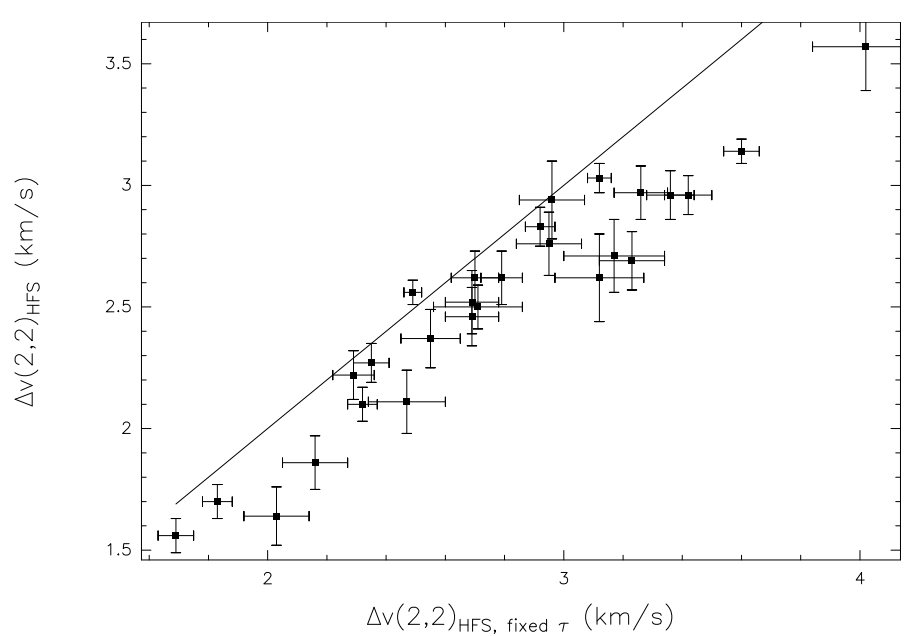

Fig. 4. Comparison of $(2,2)$ line widths determined from a hyperfine structure fit with measured $(2,2)$ optical depth against those with fixed $(2,2)$ optical depth. The solid line displays equal line widths.

change in the excitation temperature or a change in the filling factor.

The rotational temperatures range between $12 \mathrm{~K}$ and $28 \mathrm{~K}$ (see Table 3), and the mean value is $18.6 \mathrm{~K}$ with an uncertainty of $15 \%$. For the subsample described in Sect. 3.3 with detected hyperfine satellites of the $(2,2)$ line, we compared the $(2,2)$ optical depth, $\tau(2,2)_{\mathrm{HFS}}$, to the values computed with Eq. (1). The solid line in Fig. 5 indicates $\tau(2,2)=\tau(2,2)_{\text {HFS }}$. Figure 5 illustrates that the $(2,2)$ optical depths from hyperfine structure fits are larger than the calculated values, except for three sources, which might explain the offset seen in Fig. 3. The clump with the lowest $(2,2)$ optical depths of these is G331.51-0.10, for which Sevenster et al. (1997) detected OH-maser emission at $1612 \mathrm{MHz}$. The other two sources are G351.45+0.66, the bright hot core described in Sect. 3.3 that is also a known methanol maser emitting at $6.6 \mathrm{GHz}$ and $12 \mathrm{GHz}$ (Caswell et al. 1995), and G305.37+0.17, a compact HII region in the rich high-mass starforming complex G305 (Clark \& Porter 2004; Hindson et al. 2013). The dotted line indicates $\tau(2,2)=\tau(2,2)_{\mathrm{HFS}} / 2$, many sources lie between the two relations. Clumps with large fitted $(2,2)$ optical depths lie below the dotted line, they have the largest deviations between calculated and fitted $(2,2)$ optical depth. These are weak sources with the lowest $\mathrm{S} / \mathrm{Ns}$ and larger errors of the $(2,2)$ optical depth.

Because hyperfine anomalies that affect mostly the outer satellites are found in the $\mathrm{NH}_{3}$ sample in the fourth quadrant (see Sect. 3.8), the excitation temperatures of the main line and satellites are likely to be different, which might influence the estimation of the optical depth from the hyperfine structure. To test this, we compared the $(1,1)$ optical depth derived from the

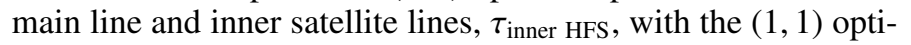
cal depth determined from all hyperfine structure lines, $\tau_{\text {all HFS }}$, including the outer satellites associated with the largest hyperfine anomalies. A fit to the two optical depths yields $\tau_{\text {inner HFS }}=$ $0.92 \tau_{\text {all HFS }}+0.17$ and a Spearman correlation test gives a coefficient of 0.86 with a $p$-value $<0.0013$. This shows that the correlation between the optical depth obtained from the main line and the inner satellites and the optical depth derived from all hyperfine structure lines is significant over $3 \sigma$. Most sources in the fourth quadrant therefore exhibit similar $\tau_{\text {inner HFS }}$ and $\tau_{\text {all HFS }}$, and the ratio $\tau_{\text {inner HFS }} / \tau_{\text {all HFS }}$ also remains constant as a function of the hyperfine anomalies.

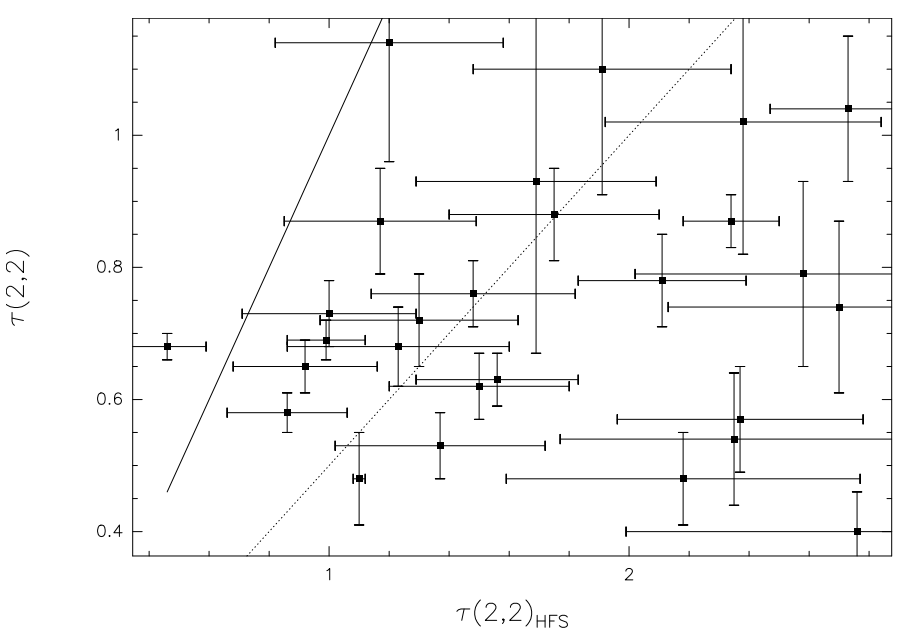

Fig. 5. $(2,2)$ optical depth calculated from the main-beam brightness temperatures of the $(1,1)$ and $(2,2)$ lines, and the $(1,1)$ optical depth is plotted against the depth derived from the hyperfine structure. The straight line indicates equal values and the dotted line $\tau(2,2)=$ $\tau(2,2)_{\mathrm{HFS}} / 2$.

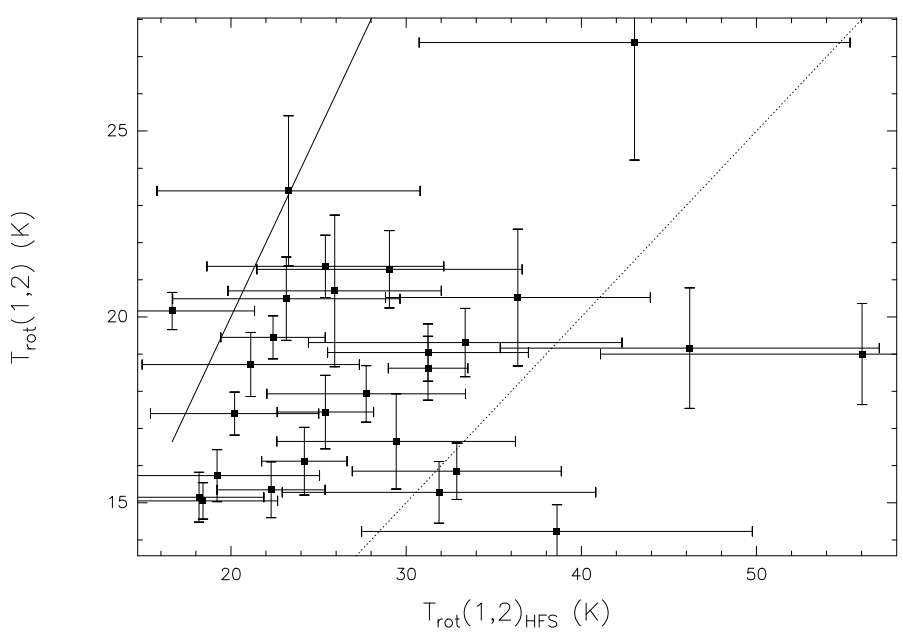

Fig. 6. Comparison of the rotational temperature between the $(1,1)$ and $(2,2)$ inversion transition using the $(2,2)$ optical depth derived from hyperfine structure fits, $T_{\text {rot }}(1,2)_{\mathrm{HFS}}$, and the temperature obtained from the calculated $(2,2)$ optical depth, $T_{\text {rot }}(1,2)$. The straight line shows equal values, while the dotted line illustrates $T_{\text {rot }}(1,2)=$ $1 / 2 T_{\text {rot }}(1,2)_{\text {HFS }}$.

The rotational temperature, $T_{\text {rot }}$, determined from Eqs. (2) and $(1)$, is compared with that using the $(2,2)$ optical depth derived from the hyperfine structure fit, $T_{\text {rot }}(1,2)_{\text {HFS }}$. Figure 6 shows that the rotational temperature with calculated $\tau(2,2)$ is mostly lower than $T_{\text {rot }}(1,2)_{\text {HFS }}$. This trend is similar to that of the $(2,2)$ optical depth in Fig. 5 and results from the difference between calculated and fitted $(2,2)$ optical depths. The relation $T_{\text {rot }}=1 / 2 T_{\text {rot }}(1,2)_{\mathrm{HFS}}$ is a lower boundary to the rotational temperature distribution of most clumps with detected $(2,2)$ hyperfine structure fits.

To test how representative clumps with $(2,2)$ hyperfine structure are for the whole southern sample, we compared their rotational temperature and $(1,1)$ optical depth. A KolmogorovSmirnov test does not contradict the assumption that the subsample and all sources in the fourth quadrant have the same rotational temperature distribution. A comparison of the $(1,1)$ optical depth shows that small optical depths are missing in the subsample with $(2,2)$ hyperfine structure, but it still covers a 
Table 4. $\mathrm{NH}_{3}$ line parameters derived from hyperfine structure fits of the $(2,2)$ inversion transition for the subsample with detected $(2,2)$ satellites.

\begin{tabular}{lcccc}
\hline \hline Name & $\tau(2,2)$ & $\begin{array}{c}\Delta v_{\text {hfs }}(2,2) \\
\left(\mathrm{km} \mathrm{s}^{-1}\right)\end{array}$ & $\begin{array}{c}T_{\text {rothhfs }} \\
(\mathrm{K})\end{array}$ & $\begin{array}{c}N_{\mathrm{NH}_{3}, \mathrm{hfs}} \\
\left(10^{14} \mathrm{~cm}^{-2}\right)\end{array}$ \\
\hline G316.81-0.06 & $1.91( \pm 0.43)$ & $2.37( \pm 0.12)$ & $25.9( \pm 6.1)$ & $50.3( \pm 13.4)$ \\
G337.92-0.48 & $1.69( \pm 0.40)$ & $2.76( \pm 0.13)$ & $43.0( \pm 12.3)$ & $49.4( \pm 22.8)$ \\
G326.64+0.61 & $0.99( \pm 0.13)$ & $3.03( \pm 0.06)$ & $22.4( \pm 3.0)$ & $34.4( \pm 4.2)$ \\
G351.45+0.66 & $0.46( \pm 0.13)$ & $2.56( \pm 0.05)$ & $16.6( \pm 4.7)$ & $19.2( \pm 2.3)$ \\
G351.58-0.35 & $2.73( \pm 0.26)$ & $2.96( \pm 0.08)$ & $24.2( \pm 2.4)$ & $89.4( \pm 9.5)$ \\
G351.77-0.54 & $2.34( \pm 0.16)$ & $3.14( \pm 0.05)$ & $31.3( \pm 2.3)$ & $77.4( \pm 7.5)$ \\
G331.88+0.06 & $2.37( \pm 0.41)$ & $2.69( \pm 0.12)$ & $32.9( \pm 6.0)$ & $71.4( \pm 17.8)$ \\
G332.09-0.42 & $1.17( \pm 0.32)$ & $2.22( \pm 0.10)$ & $23.2( \pm 6.5)$ & $30.6( \pm 8.3)$ \\
G305.37+0.17 & $1.20( \pm 0.38)$ & $2.94( \pm 0.16)$ & $23.3( \pm 7.5)$ & $42.9( \pm 13.4)$ \\
G328.26-0.53 & $1.50( \pm 0.30)$ & $2.97( \pm 0.11)$ & $27.7( \pm 5.7)$ & $48.5( \pm 11.8)$ \\
G327.29-0.58 & $0.92( \pm 0.24)$ & $2.27( \pm 0.08)$ & $25.4( \pm 6.8)$ & $21.1( \pm 6.1)$ \\
G329.03-0.20 & $1.23( \pm 0.37)$ & $2.52( \pm 0.13)$ & $19.2( \pm 5.9)$ & $39.8( \pm 8.3)$ \\
G333.02+0.77 & $0.86( \pm 0.20)$ & $2.83( \pm 0.08)$ & $20.2( \pm 4.8)$ & $30.0( \pm 5.5)$ \\
G333.13-0.56 & $2.11( \pm 0.28)$ & $2.96( \pm 0.10)$ & $22.3( \pm 3.1)$ & $72.2( \pm 9.4)$ \\
G336.02-0.83 & $2.70( \pm 0.57)$ & $2.71( \pm 0.15)$ & $46.2( \pm 10.8)$ & $77.4( \pm 29.1)$ \\
G316.77-0.02 & $1.00( \pm 0.29)$ & $2.62( \pm 0.11)$ & $21.1( \pm 6.2)$ & $31.2( \pm 7.6)$ \\
G337.92-0.46 & $1.30( \pm 0.33)$ & $2.62( \pm 0.11)$ & $29.1( \pm 7.6)$ & $35.7( \pm 11.6)$ \\
G342.71+0.13 & $1.56( \pm 0.27)$ & $2.10( \pm 0.07)$ & $31.3( \pm 5.7)$ & $37.8( \pm 9.3)$ \\
G343.50+0.02 & $2.18( \pm 0.59)$ & $1.86( \pm 0.11)$ & $31.9( \pm 9.0)$ & $42.4( \pm 16.0)$ \\
G305.24+0.26 & $1.37( \pm 0.35)$ & $2.46( \pm 0.12)$ & $33.4( \pm 9.0)$ & $36.1( \pm 13.4)$ \\
G351.78-0.52 & $1.48( \pm 0.34)$ & $1.70( \pm 0.07)$ & $18.4( \pm 4.3)$ & $39.8( \pm 5.6)$ \\
G331.55-0.07 & $2.35( \pm 0.58)$ & $2.62( \pm 0.18)$ & $56.0( \pm 14.9)$ & $89.3( \pm 38.6)$ \\
G354.62+0.47 & $1.10( \pm 0.02)$ & $2.50( \pm 0.09)$ & $25.4( \pm 2.8)$ & $24.5( \pm 3.9)$ \\
G347.63+0.15 & $2.38( \pm 0.46)$ & $3.57( \pm 0.18)$ & $36.4( \pm 7.6)$ & $104.5( \pm 31.9)$ \\
G351.74-0.58 & $1.75( \pm 0.35)$ & $1.56( \pm 0.07)$ & $18.2( \pm 3.7)$ & $41.5( \pm 5.2)$ \\
G345.72+0.82 & $2.76( \pm 0.77)$ & $1.64( \pm 0.12)$ & $38.6( \pm 11.2)$ & $49.1( \pm 21.1)$ \\
G347.97-0.43 & $2.58( \pm 0.56)$ & $2.11( \pm 0.13)$ & $29.4( \pm 6.8)$ & $55.7( \pm 16.6)$ \\
\hline & & & &
\end{tabular}

wide range from $\sim 1$ to 5 and is not strongly skewed to sources exhibiting high optical depths.

We present the rotational temperature and column density obtained from the measured $(2,2)$ optical depth in Table 4 . We estimate a ratio of $T_{\mathrm{rot}}(1,2)_{\mathrm{HFS}}$ to $T_{\mathrm{rot}}$ of $1.56 \pm 0.5$ and a ratio of the $\mathrm{NH}_{3}$ column density derived using the $(2,2)$ optical depth from the hyperfine structure fit to that determined from the $(1,1)$ optical depth and the line temperature ratio of $1.56 \pm 0.64$.

These factors are not used to correct the rotational temperature and column density of the whole sample in the fourth quadrant, to be consistent with our method in the first quadrant (Wienen et al. 2012). Moreover, the $(2,2)$ hyperfine structure could only be detected for a small subsample of 27 sources, resulting in a large scatter of the data. The hyperfine structure fit with the $(2,2)$ optical depth derived from the $(1,1)$ optical depth and line temperature ratio leads to an underestimation of the rotational temperature and column density.

\subsection{Comparison of $\mathrm{NH}_{3}$ line parameters in the first and fourth quadrant}

We used the same assumptions and equations to calculate the source-averaged $\mathrm{NH}_{3}$ column density and the kinetic temperature from the rotational temperature of the ATLASGAL sources in the fourth quadrant as in the first quadrant, which are given in Wienen et al. (2012). Figure 7 displays the kinetic temperature plotted against the logarithm of the $\mathrm{NH}_{3}$ column density for the southern sources as red points and the northern sample in black. We obtained kinetic temperatures of the southern clumps in a similar range as those of the northern $\mathrm{NH}_{3}$ sample from $13 \mathrm{~K}$ to $40 \mathrm{~K}$ with an uncertainty of $24 \%$. The median kinetic temperature of clumps in the first quadrant is $19 \mathrm{~K}$, which is slightly lower than the peak of $23 \mathrm{~K}$ of the southern sources in Fig. 7. However, the sample in the first quadrant contains sources with lower peak fluxes, decreasing to $0.4 \mathrm{Jy} / \mathrm{beam}$, than that in the fourth quadrant. The median kinetic temperature of northern clumps with a peak flux density above $1.2 \mathrm{Jy} /$ beam is $\sim 22 \mathrm{~K}$ and agrees with that of the sources in the fourth quadrant. Values of the $\mathrm{NH}_{3}$ column density lie between $5 \times 10^{14} \mathrm{~cm}^{-2}$ and $7 \times 10^{15} \mathrm{~cm}^{-2}$, the peak at $2 \times 10^{15} \mathrm{~cm}^{-2}$ is the same as obtained for the northern clumps. We show only sources with small errors of the line parameters $(<\sim 50 \%)$ on the correlation plots. Figure 8 presents the distribution of the logarithm of the $\mathrm{NH}_{3}$ abundance for the sample in the first quadrant as a solid black curve and for sources in the fourth quadrant as a dashed red curve. The comparison shows a consistent range of the abundance for the two subsamples. The slightly higher abundance peak of the northern sample observed with the Effelsberg telescope might result from a smaller beam width tracing higher abundances towards the central parts of the clumps. 

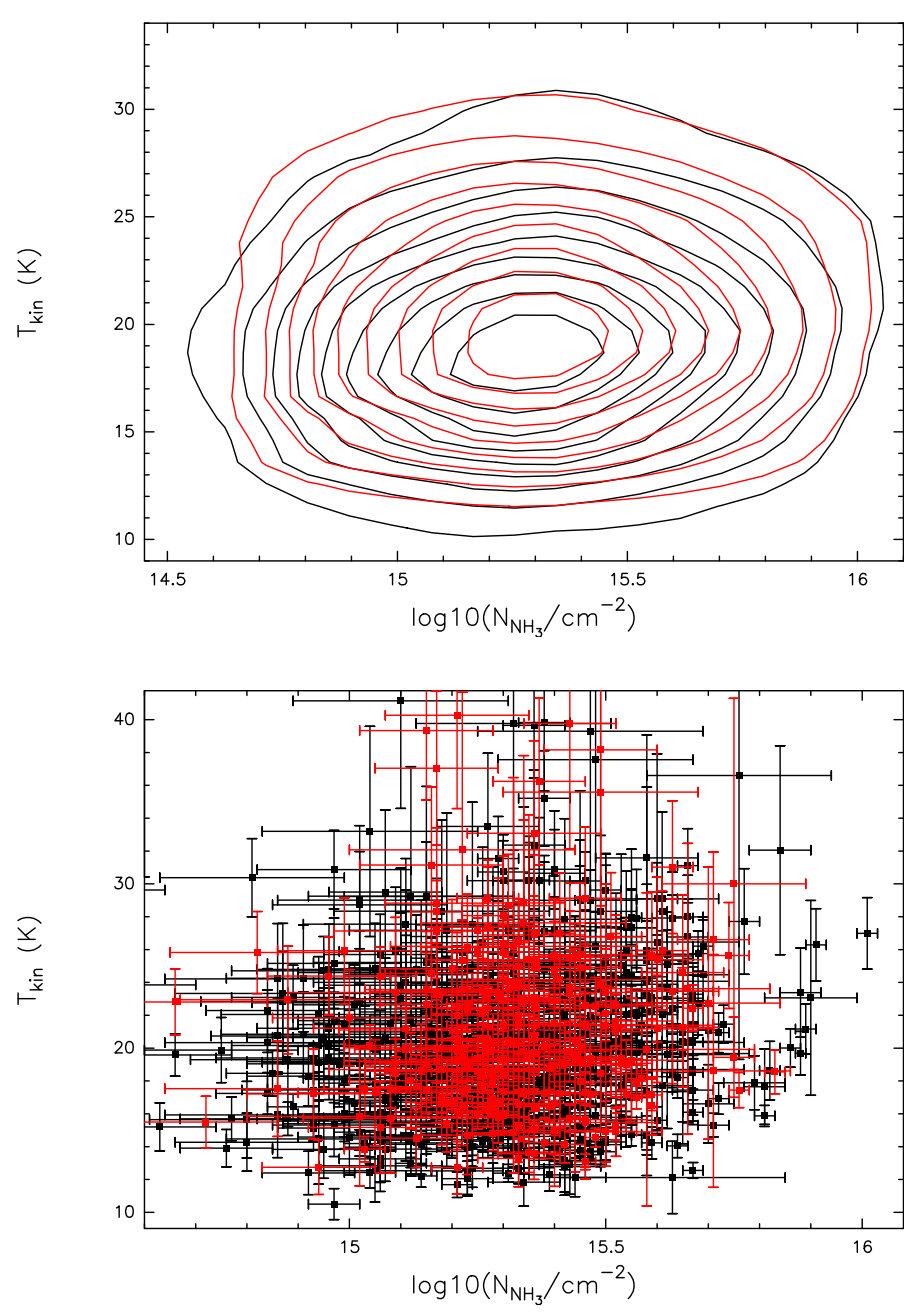

Fig. 7. Kinetic temperature plotted against the logarithm of the $\mathrm{NH}_{3}$ column density. Northern ATLASGAL sources are shown in black and the southern sample in red. For the contour plot in the upper panel, we counted the number of sources in each column density bin of 0.5 dex and in each kinetic temperature bin of $3 \mathrm{~K}$. The contours illustrate $10 \%$ to $90 \%$ in steps of $10 \%$ of the peak source number per bin. These levels are shown in all contour plots in this article.

\subsection{Variation of $\mathrm{NH}_{3}$ line parameters}

The longitude and radial velocity of a source determine a unique galactocentric radius using a model of the Galactic rotation curve (e.g. Brand \& Blitz 1993). We analysed trends of physical parameters obtained from $\mathrm{NH}_{3}$ inversion transitions within the inner Galaxy. We divided galactocentric radii of the $\mathrm{NH}_{3}$ subsamples in the first and fourth quadrant of between $3 \mathrm{kpc}$ and $8 \mathrm{kpc}$ into bins of width $1 \mathrm{kpc}$ and investigated the distribution of the means of each bin. The rotational temperature, calculated with Eq. (2), shows a flat distribution for the northern and southern subsamples with mean values of between $16 \mathrm{~K}$ and $23 \mathrm{~K}$, which is consistent with kinetic temperatures of sources from the BGPS (Bolocam Galactic Plane Survey; Aguirre et al. 2011) within the inner Galaxy observed in $\mathrm{NH}_{3}$ (Dunham et al. 2011). We also inspected the influence of the heliocentric distance on the distribution of the rotational temperature and $\mathrm{NH}_{3}(1,1)$ line width (see Sect. 3.3), but no dependence was found. Furthermore, we computed the $\mathrm{H}_{2}$ column density via Eq. (11) in Wienen et al. (2012) using the beam width of 61" of the Parkes telescope. We smoothed the ATLASGAL maps to the resolution of the Parkes telescope and extracted the peak flux of the clumps. The mean

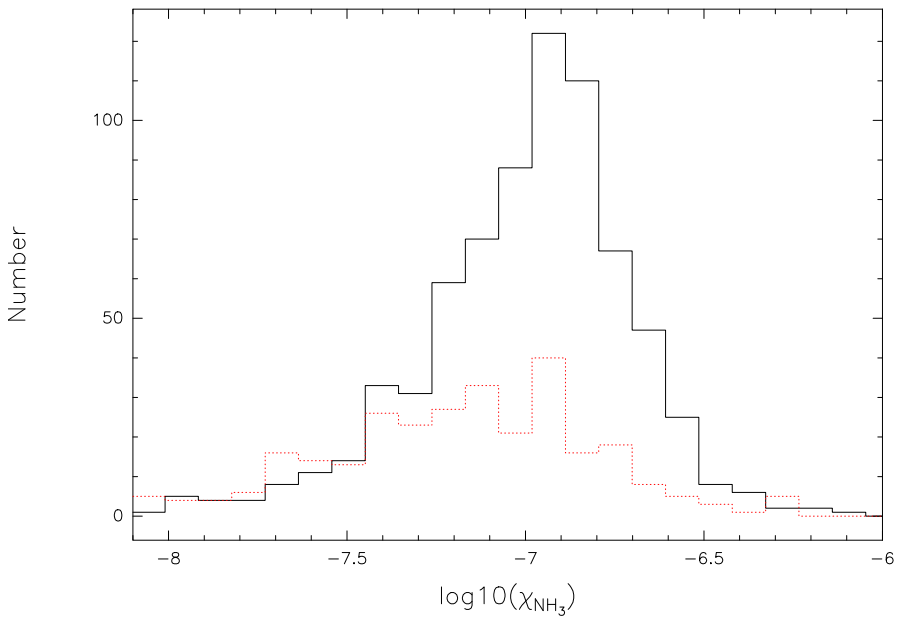

Fig. 8. Histogram of the logarithm of the $\mathrm{NH}_{3}$ abundance for sources in the first quadrant are plotted as a solid black curve and for clumps in the fourth quadrant as a dashed red curve.

values of the $\mathrm{H}_{2}$ column density in the first and fourth quadrants lie between $10^{22}$ and $4 \times 10^{22} \mathrm{~cm}^{-2}$ within galactocentric radii from 3 to $8 \mathrm{kpc}$, which is similar to the mean $\mathrm{H}_{2}$ column density of the BGPS sample. Moreover, the $(1,1)$ line widths, exhibiting mean values between $\sim 2$ and $4 \mathrm{~km} \mathrm{~s}^{-1}$, are also approximately constant within a $3 \sigma$ uncertainty for the northern and southern subsamples. This shows that clumps in the spiral arms are not hotter or more turbulent, which indicates that the ratio of starforming to quiescent clumps is the same in the spiral arms and the interarm regions. This is consistent with the investigations of Eden et al. (2013) and Battisti \& Heyer (2014), who found no changes in clump formation efficiency with different environments located in the Galactic plane.

The analysis of the $\mathrm{NH}_{3}$ abundance as a function of galactocentric radius towards BGPS sources by Dunham et al. (2011) shows a decreasing trend. We also determined the $\mathrm{NH}_{3}$ abundance of ATLASGAL sources in the first and fourth quadrant as described in Wienen et al. (2012). While we used the same relation to calculate the $\mathrm{H}_{2}$ column density as Dunham et al. (2010), we derived a source-averaged $\mathrm{NH}_{3}$ column density similar as in Wienen et al. (2012), and Dunham et al. (2010) reported a beamaveraged $\mathrm{NH}_{3}$ column density. Because our $\mathrm{NH}_{3}$ abundances are affected by the unknown filling factor of the sources as described in Wienen et al. (2012), we obtain higher values from $\sim 10^{-6}$ to $10^{-8}$ than the range determined by Dunham et al. (2011) between $10^{-7}$ and $10^{-9}$. The upper panel of Fig. 9 shows the logarithm of the $\mathrm{NH}_{3}$ abundance plotted against the galactocentric radius. The whole sample is illustrated as black points and the mean of each bin in galactocentric radius as red points. The distribution is fitted by the relation

$\chi_{\mathrm{NH}_{3}}=10^{-6.70 \pm 0.04} 10^{-0.063 \pm 0.007 R_{\text {gal }}}$.

This reveals a decrease in $\mathrm{NH}_{3}$ abundance of $-0.063 \pm$ $0.007 \mathrm{dex} / \mathrm{kpc}$. Using the $\mathrm{NH}_{3}$ abundance of BGPS sources (Dunham et al. 2011) only within galactocentric radii between $\sim 1$ and $8 \mathrm{kpc}$, we obtain a gradient of $-0.067 \pm 0.016 \mathrm{dex} / \mathrm{kpc}$, which is slightly steeper than our value, but agrees within $3 \sigma$. While the ATLASGAL sample lies within galactocentric radii from 1.2 to $8.4 \mathrm{kpc}$, Dunham et al. (2011) added the Gemini OB1 molecular cloud from the outer Galaxy, which lowers their gradient in the $\mathrm{NH}_{3}$ abundance to $-0.096 \mathrm{dex} / \mathrm{kpc}$. They considered varying dust properties such as a changing gas-to-dust ratio, different dust temperatures, or different metallicities in the 

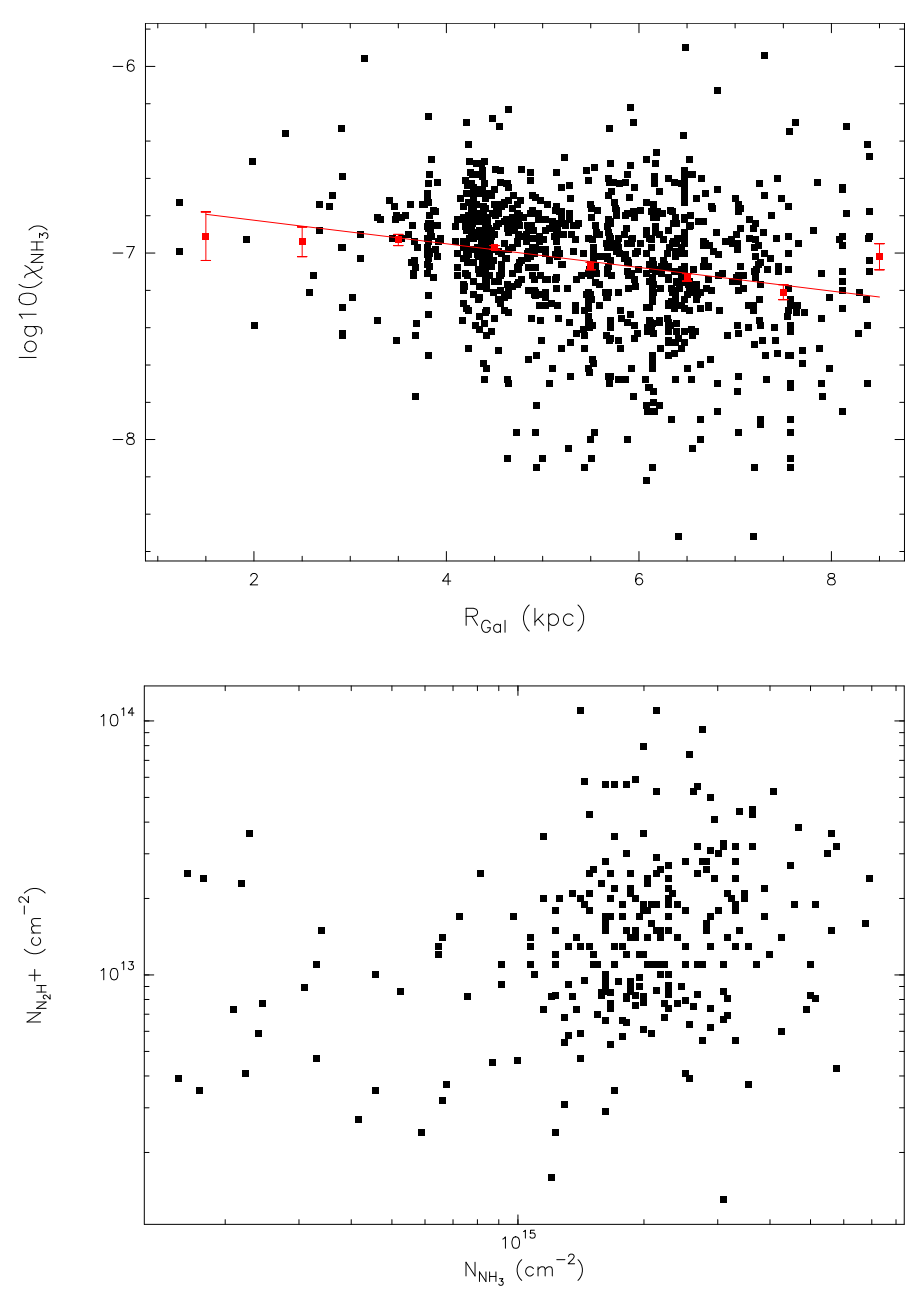

Fig. 9. Dependence of the logarithm of the $\mathrm{NH}_{3}$ abundance on the galactocentric radius (upper panel). The whole sample is displayed in black, while the mean of each bin in galactocentric radius is indicated in red. The straight line shows a fit to the binned data. The correlation plot of the $\mathrm{NH}_{3}$ and $\mathrm{N}_{2} \mathrm{H}^{+}$column density is illustrated in the lower panel.

Galaxy as explanations for this trend. In addition, they suggested that the decreasing nitrogen abundance with rising galactocentric radii (e.g. Shaver et al. 1983; Rolleston et al. 2000) is likely to reduce the $\mathrm{NH}_{3}$ formation. Shaver et al. (1983) determined a gradient in the nitrogen abundance of $-0.09 \pm 0.015 \mathrm{dex} / \mathrm{kpc}$ mainly in the outer Galaxy, but it is unclear if the decrease still exists in the inner Galaxy. We only obtain a gradient for the ATLASGAL sample when we include $\mathrm{NH}_{3}$ abundances at the innermost galactocentric radii between 1 and $4 \mathrm{kpc}$. If weaker $\mathrm{NH}_{3}$ formation resulted from a reduced amount of nitrogen, we would expect an even steeper decrease in $\mathrm{N}_{2} \mathrm{H}^{+}$abundance, consisting of two nitrogen atoms, than of $\mathrm{NH}_{3}$. This would result in a decreasing trend of the $\mathrm{N}_{2} \mathrm{H}^{+} / \mathrm{NH}_{3}$ column density ratio as a function of galactocentric radius. We measured the $\mathrm{N}_{2} \mathrm{H}^{+}(1-0)$ line using the Mopra telescope for a southern ATLASGAL subsample of 293 sources observed in $\mathrm{NH}_{3}$. The $\mathrm{N}_{2} \mathrm{H}^{+}(1-0)$ column density was calculated using (Benson et al. 1998)

$N_{\mathrm{N}_{2} \mathrm{H}^{+}}=\frac{3.3 \times 10^{11} \tau \Delta v_{\mathrm{N}_{2} \mathrm{H}^{+}} T_{\text {kin }}}{1-\exp \left(-4.47 / T_{\text {kin }}\right)}$,

with the optical depth of the $\mathrm{N}_{2} \mathrm{H}^{+}(1-0)$ line, $\tau$, the $\mathrm{N}_{2} \mathrm{H}^{+}$line width, $\Delta v_{\mathrm{N}_{2} \mathrm{H}^{+}}$, and the kinetic temperature derived from $\mathrm{NH}_{3}$ observations, $T_{\text {kin }}$. While Benson et al. (1998) used in this equation the excitation temperature, it can be low due to an additional small beam-filling factor or sub-thermal excitation. To be consistent with our $\mathrm{NH}_{3}$ analysis, we used instead the kinetic temperature in Eq. (5) to estimate the $\mathrm{N}_{2} \mathrm{H}^{+}$column density. Systematic errors are introduced by replacing the excitation temperature with the kinetic temperature. To estimate these, we computed with RADEX (van der Tak et al. 2007) the expected excitation temperature for a range of $\mathrm{H}_{2}$ densities using typical column densities and kinetic temperatures of our sample. For a density of $10^{5} \mathrm{~cm}^{-3}$ similar to the median of the ATLASGAL sample (Wienen et al. 2015), the $\mathrm{NH}_{3}$ column density would be underestimated by $37 \%$, while for higher densities of $10^{6} \mathrm{~cm}^{-3}$, more appropriate to regions dominated by the more compact $\mathrm{N}_{2} \mathrm{H}^{+}$ emission, we would overestimate the column density by a factor of 1.45. The comparison of the $\mathrm{N}_{2} \mathrm{H}^{+}$and $\mathrm{NH}_{3}$ column density is presented in the lower panel of Fig. 9, which shows higher $\mathrm{NH}_{3}$ column densities ranging from $1.5 \times 10^{14}$ to $7 \times 10^{15} \mathrm{~cm}^{-2}$ than $\mathrm{N}_{2} \mathrm{H}^{+}$column densities between $6 \times 10^{12}$ and $1.7 \times 10^{15} \mathrm{~cm}^{-2}$ and a trend of increasing $\mathrm{N}_{2} \mathrm{H}^{+}$column density with rising $\mathrm{NH}_{3}$ column density. The logarithm of the $\mathrm{N}_{2} \mathrm{H}^{+} / \mathrm{NH}_{3}$ column density ratio is illustrated in Fig. 10, which indicates an approximately constant distribution fitted by

$N_{\mathrm{N}_{2} \mathrm{H}^{+}} / N_{\mathrm{NH}_{3}}=10^{-1.65 \pm 0.12} 10^{0.039 \pm 0.02 R}$ gal .

We performed a t-test to investigate whether the slope of the distribution is equal to 0 . This is rejected if the $p$-value is lower than the assumed significance level of 0.05 . Our t-test result gives a $p$-value of 0.11 and therefore does not contradict the assumption that the distribution can be fitted by a function with a slope of 0 . This is consistent with the formation of $\mathrm{N}_{2} \mathrm{H}^{+}$and $\mathrm{NH}_{3}$ from $\mathrm{N}_{2}$ in most gas-phase models. For the production of $\mathrm{N}_{2} \mathrm{H}^{+}$, the reaction of $\mathrm{CH}$ with $\mathrm{N}$ forms $\mathrm{CN}$, which reacts with $\mathrm{N}$ and gives $\mathrm{N}_{2}$. This reacts with $\mathrm{H}_{3}^{+}$and produces $\mathrm{N}_{2} \mathrm{H}^{+}$(Nejad et al. 1990; Womack et al. 1992). $\mathrm{NH}_{3}$ can be formed through the reaction of $\mathrm{He}^{+}$with $\mathrm{N}_{2}$, which gives $\mathrm{N}^{+}$. Subsequent hydrogenation reactions with $\mathrm{H}_{2}$ result in the formation of $\mathrm{NH}^{+}, \mathrm{NH}_{2}^{+}, \mathrm{NH}_{3}^{+}$, and $\mathrm{NH}_{4}^{+}$. The recombination of $\mathrm{NH}_{4}^{+}$with an electron produces $\mathrm{NH}_{3}$ (Le Bourlot 1991). Consequently, the $\mathrm{NH}_{3}$ and $\mathrm{N}_{2} \mathrm{H}^{+}$abundances are found to be approximately proportional to $\mathrm{N}_{2}$ in models of prestellar cores in dark clouds (Hily-Blant et al. 2010), which would lead us to expect a similar trend of the $\mathrm{N}_{2} \mathrm{H}^{+}$and $\mathrm{NH}_{3}$ column density with galactocentric radius. Moreover, $\mathrm{NH}_{3}$ can also be formed on dust grains and return to the gas through evaporation or sputtering in shocks when the temperature rises (e.g. Aikawa et al. 2012). This formation process is important close to protostars and in hot cores and is therefore likely to occur in ATLASGAL sources, which are in a more evolved phase of high-mass star formation and exhibit high rotational temperatures and broad line widths.

The decrease in the $\mathrm{NH}_{3}$ abundance across the Galaxy as shown in Dunham et al. (2011) and Fig. 9 might result from a lowered nitrogen abundance, but a larger number of nitrogen atoms within a certain molecule might not be related to an enhanced decrease of its abundance.

\section{7. $\mathrm{NH}_{3}(3,3)$ masers}

The inversion of the $\mathrm{NH}_{3}(3,3)$ level is explained with a pumping process of the $(3,3)$ maser by Guilloteau et al. (1983) and Walmsley \& Ungerechts (1983). Depending on the nuclear spin configuration of the hydrogen atoms, we distinguish between two species of the ammonia molecule: all hydrogen spins are parallel in ortho- $\mathrm{NH}_{3}$ with $K=3 n$, and one pair is anti-parallel in para- $\mathrm{NH}_{3}$ with $K \neq 3 n$. Radiative transitions follow the selection rule $\Delta K=0$ and collisional transitions $\Delta K=3 n$, which 


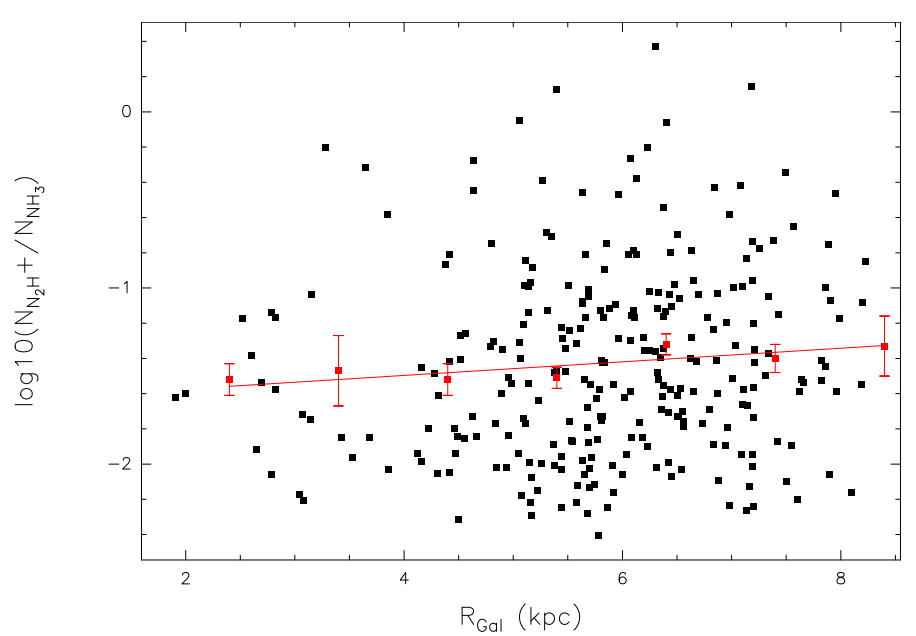

Fig. 10. Distribution of the $\mathrm{N}_{2} \mathrm{H}^{+} / \mathrm{NH}_{3}$ column density ratio within the inner Galaxy. The whole sample is shown as black points and the mean of each bin in galactocentric radius as red points.

cannot change the spin orientation and does therefore not transfer between ortho- and para- $\mathrm{NH}_{3}$. Collisions between levels of different parity are favoured. As the lower $(3,3)^{-}$state has the same parity as the $(0,0)^{-}$level, the upper state $(3,3)^{+}$is collisionally connected with the $(2,0)^{-}$and $(0,0)^{-}$states. The lower $(3,3)^{-}$level is depopulated through $\Delta K=3$ collisional transitions to the $(1,0)^{+}$state, which decays through radiation to the $(0,0)^{-}$level. The inversion of the $(3,3)$ line results from the population of the upper level by collisions with the $(0,0)^{-}$ state. Walmsley \& Ungerechts (1983) estimated an $\mathrm{H}_{2}$ density of $7000 \mathrm{~cm}^{-3}$, above which this pumping process is expected. For densities higher than $10^{5} \mathrm{~cm}^{-3}$, the $K=0$ levels become populated, can thermalize, and the maser is quenched.

$\mathrm{NH}_{3}$ (3,3) masers have been detected in several high-mass star-forming regions such as DR 21(OH) (Mangum \& Wootten 1994), NGC 6334I (Kraemer \& Jackson 1995), IRAS 20126+4104 (Zhang et al. 1999), G5.89-0.39 (Hunter et al. 2008), G23.33-0.30 (Walsh et al. 2011), G30.7206-00.0826 (Urquhart et al. 2011), and G35.03+0.35 (Brogan et al. 2011). We searched for $\mathrm{NH}_{3}(3,3)$ masers in the ATLASGAL sample using the following criteria:

1. We searched for sources in which the noise in the Gaussian fit of the $(3,3)$ line is higher than the noise of the baseline. Such clumps have a $(3,3)$ line profile that deviates from a Gaussian.

2. The $(3,3)$ line has a Gaussian profile, the noise of the baseline is therefore higher than or similar to the error in the fitted $(3,3)$ line, but the $(3,3)$ line width is smaller than the thermal $(1,1)$ and $(2,2)$ line widths. To ensure that we selected spectra with high $\mathrm{S} / \mathrm{N}$, the intensity of the $(3,3)$ line must be higher than $5 \sigma$, where $\sigma$ is the noise of the baseline.

Taking sources with the largest deviation of the error in the Gaussian fit from the noise of the baseline, $\sigma_{\text {Gauss }} / \sigma_{\text {base }}>1.3$, into account, we find three $\mathrm{NH}_{3}(3,3)$ masers. Examining the largest deviation of the noise of the baseline from the error in the fit, $\sigma_{\text {base }} / \sigma_{\text {Gauss }}>1.6$, and the smallest line-width ratio, $\Delta v(3,3) / \Delta v(1,1)<1.2$, results in an additional five masers with an $\mathrm{S} / \mathrm{N}$ of the $(3,3)$ line higher than $5 \sigma$. We give two examples with the largest $\sigma_{\text {Gauss }} / \sigma_{\text {base }}$ in Fig. 11: The $(3,3)$ line of $\mathrm{G} 333.02+0.77$ shows an additional peak at a sligthly lower velocity than that of the $(3,3)$ thermal emission. This source is also known as IRAS 16115-4941, where Scalise et al. (1989)
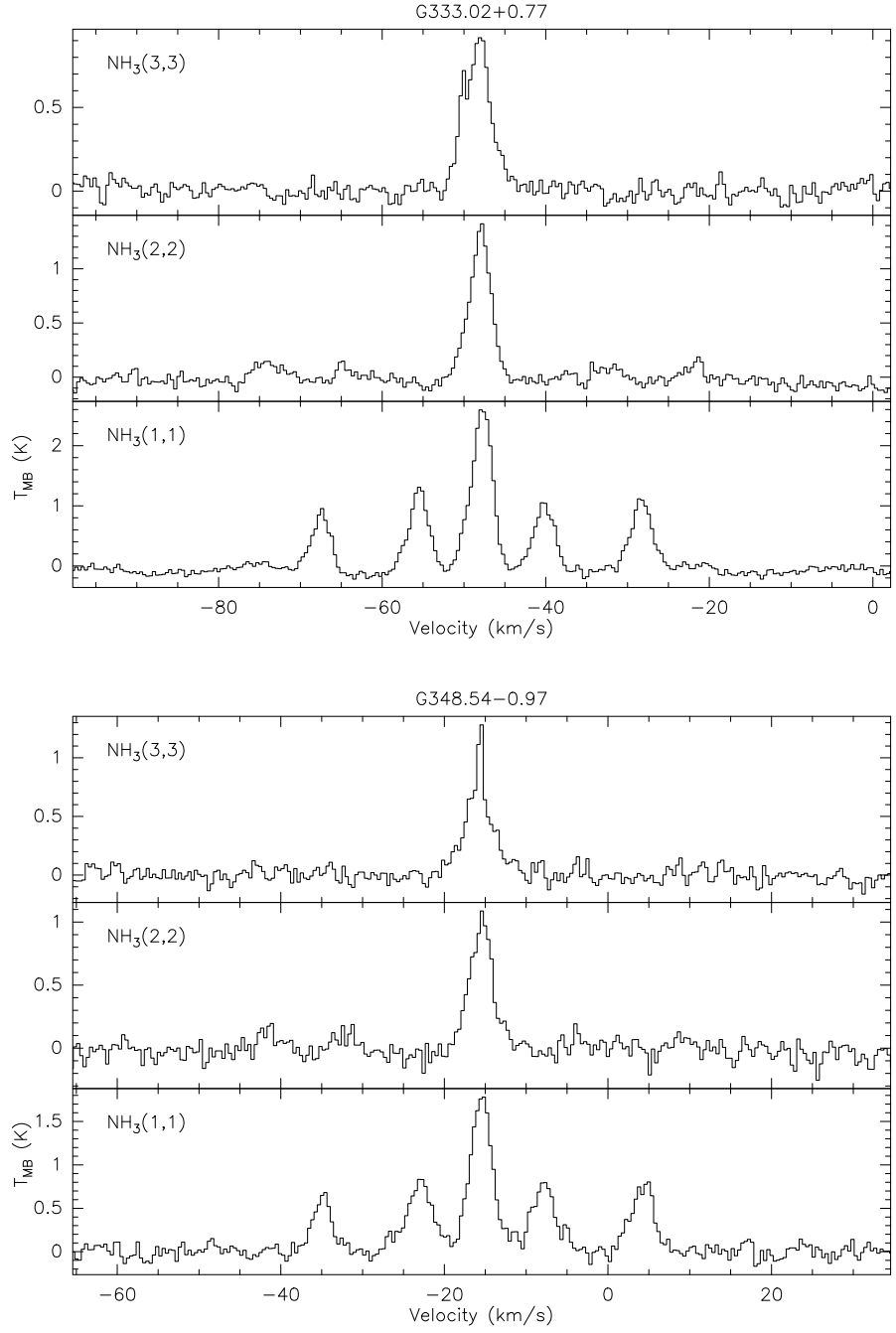

Fig. 11. $\mathrm{NH}_{3}(1,1)$ to $(3,3)$ inversion transitions of two ATLASGAL sources with a $(3,3)$ line profile that deviates from a Gaussian fit and indicates a maser line.

observed $\mathrm{H}_{2} \mathrm{O}$ maser emission. Urquhart et al. (2007) used highresolution radio continuum observations at 3.6 and $6 \mathrm{~cm}$ to identify G333.02+0.77 as an irregular/multi-peaked UCHIIR. Anderson et al. (2011) observed hydrogen radio recombination lines at $9 \mathrm{GHz}$ towards G348.54-0.97, which is identified as an HII region based on its association with the radio continuum at $20 \mathrm{~cm}$ and $24 \mu \mathrm{m}$ emission. Their investigation of GLIMPSE maps shows a surrounding layer of $8 \mu \mathrm{m}$ emission, which characterizes the source as a bubble. Because G348.54-0.97 is associated with methanol masers (Caswell et al. 1995; Pestalozzi et al. 2005) and CS (2-1) emission (Bronfman et al. 1996), it is likely to be a young HII region.

\subsection{Measuring anomalies in the $\mathrm{NH}_{3}(1,1)$ quadrupole hyperfine structure}

It has been suggested that deviations in the intensity ratios of the $\mathrm{NH}_{3}(1,1)$ inner and outer satellite lines to the main line from a symmetric distribution in local thermal equilibrium (LTE) can be caused by two processes: a nonthermal excitation of the $(1,1)$ hyperfine levels as described by Matsakis et al. (1977), or systematic motion as suggested by Park (2001). A large portion of the $\mathrm{NH}_{3}$ sample shows such hyperfine anomalies; an example spectrum with an overlaid fit indicating the expected symmetric 
Table 5. Main-beam brightness temperature ratios of the $\mathrm{NH}_{3}(1,1)$ quadrupole hyperfine structure components.

\begin{tabular}{lllll}
\hline \hline Name & $\alpha$ & $\beta$ & $\gamma$ & $\sigma$ \\
\hline G300.72+1.20 & $1.26( \pm 1.47)$ & $1.10( \pm 0.80)$ & $1.16( \pm 0.74)$ & 0.05 \\
G300.82+1.15 & $0.87( \pm 0.33)$ & $0.77( \pm 0.23)$ & $0.82( \pm 0.19)$ & 0.04 \\
G300.91+0.88 & $1.29( \pm 0.42)$ & $0.98( \pm 0.24)$ & $1.10( \pm 0.21)$ & 0.06 \\
G300.97+1.15 & $1.48( \pm 0.89)$ & $1.06( \pm 0.51)$ & $1.23( \pm 0.46)$ & 0.03 \\
G301.01+1.11 & $1.31( \pm 0.51)$ & $0.88( \pm 0.26)$ & $1.05( \pm 0.25)$ & 0.05 \\
G301.12+0.96 & $1.48( \pm 0.30)$ & $0.98( \pm 0.15)$ & $1.17( \pm 0.14)$ & 0.05 \\
G301.12+0.98 & $1.01( \pm 0.13)$ & $0.95( \pm 0.10)$ & $0.98( \pm 0.08)$ & 0.05 \\
G301.14+1.01 & $1.29( \pm 0.28)$ & $0.93( \pm 0.15)$ & $1.07( \pm 0.14)$ & 0.05 \\
G301.68+0.25 & $0.66( \pm 0.17)$ & $1.01( \pm 0.21)$ & $0.83( \pm 0.14)$ & 0.06 \\
G301.74+1.10 & $1.15( \pm 0.16)$ & $1.01( \pm 0.12)$ & $1.07( \pm 0.09)$ & 0.02 \\
G301.81+0.78 & $0.06( \pm 0.63)$ & $0.56( \pm 0.45)$ & $0.37( \pm 0.36)$ & 0.05 \\
G302.39+0.28 & $1.95( \pm 0.68)$ & $1.27( \pm 0.32)$ & $1.54( \pm 0.31)$ & 0.03 \\
G304.20+1.34 & $1.63( \pm 0.72)$ & $0.79( \pm 0.28)$ & $1.10( \pm 0.29)$ & 0.04 \\
G304.76+1.34 & $1.27( \pm 0.37)$ & $1.04( \pm 0.21)$ & $1.13( \pm 0.19)$ & 0.05 \\
\hline
\end{tabular}

Notes. Errors are given in parentheses. $\sigma$ denotes the noise of the spectrum; weak hyperfine structure lines with brightness temperatures lower than $\sigma$ are not used. The full table is available at the CDS.

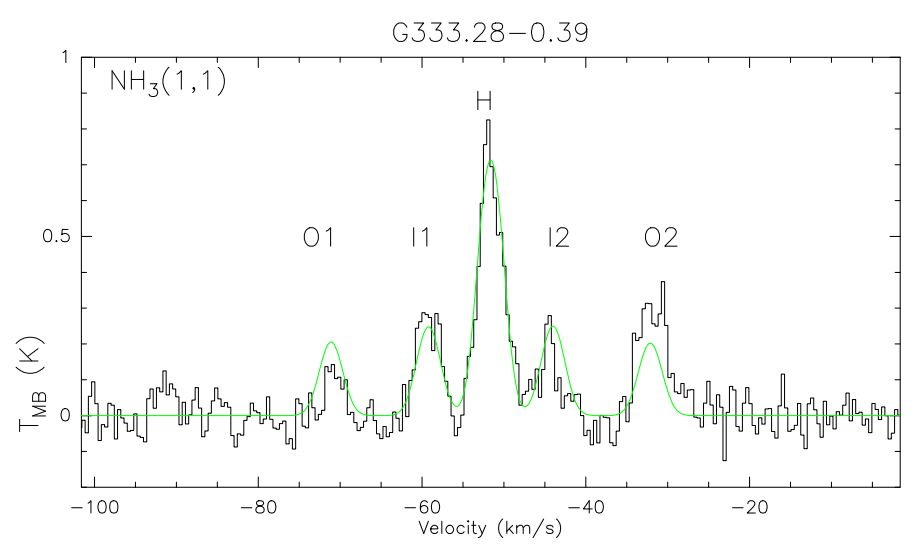

Fig. 12. Example of observed $\mathrm{NH}_{3}(1,1)$ hyperfine structure lines, the outer satellite lines are denoted by $\mathrm{O} 1$ and $\mathrm{O} 2$, the inner hyperfine lines by $\mathrm{I} 1$ and $\mathrm{I} 2$, and the main line by $\mathrm{H}$. The $\mathrm{NH}_{3}$ hyperfine structure fit assuming LTE conditions is overlaid in green, which displays equal intensities of $\mathrm{O} 1$ and $\mathrm{O} 2$ as well as of $\mathrm{I} 1$ and $\mathrm{I} 2$. The outer satellite lines of the observed spectrum deviate from this prediction.

satellite ratios is given in Fig. 12. The outer satellites are denoted by $\mathrm{O} 1$ and $\mathrm{O} 2$, the inner satellites by $\mathrm{I} 1$ and $\mathrm{I} 2$, and the main hyperfine line by $\mathrm{H}$. The intensities of the outer satellite lines are decreased $(\mathrm{O} 1)$ or enhanced $(\mathrm{O} 2)$ with respect to the predicted spectrum.

To analyse which of the two suggestions leads to our observed hyperfine anomalies, we examined the ratios of the inner and outer satellite lines as described by Longmore et al. (2007). If the selective radiative trapping in non-LTE conditions creates the anomalies, the intensity of one outer satellite (O2 in Fig. 12) will be higher than that of the other outer hyperfine line (O1 in Fig. 12). In contrast, systematic motion can produce asymmetries in the outer and inner hyperfine structure lines. Hence, the satellites $\mathrm{O} 1$ and $\mathrm{I} 1$ are stronger than $\mathrm{I} 2$ and $\mathrm{O} 2$ for infall as a result of core contraction, while $\mathrm{I} 2$ and $\mathrm{O} 2$ have increased intensities compared to $\mathrm{O} 1$ and $\mathrm{I} 1$ for outflow in expanding sources, as shown by Park (2001). We adapted the technique from Longmore et al. (2007) and fitted individual Gaussian profiles to the five hyperfine structure lines to compute their main-beam brightness temperatures. Longmore et al. (2007) measured the hyperfine anomalies by calculating the ratio

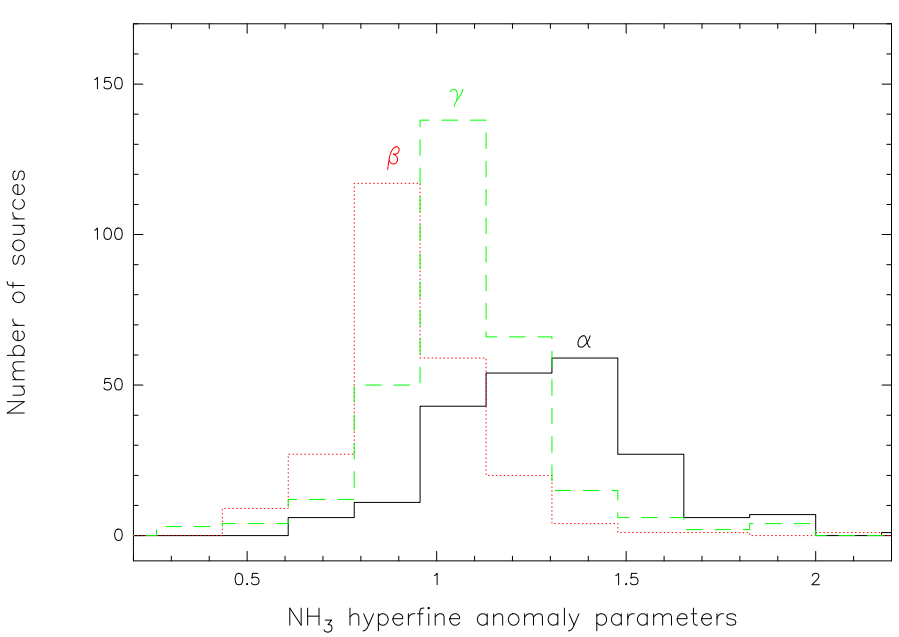

Fig. 13. Number distribution of the $\mathrm{NH}_{3}$ hyperfine anomaly parameters $\alpha$ as the solid black curve, $\beta$ as the dotted red curve, and $\gamma$ as the dashed green curve.

of the temperatures of the different hyperfine components, where

$\alpha=\frac{\mathrm{O} 2}{\mathrm{O} 1}$

$\beta=\frac{\mathrm{I} 2}{\mathrm{I} 1}$,

$\gamma=\frac{\mathrm{I} 2+\mathrm{O} 2}{\mathrm{O} 1+\mathrm{I} 1}$.

These parameters of our $\mathrm{NH}_{3}$ observations are given in Table 5 with the errors calculated from Gaussian error propagation. We also add the noise of the spectrum, which is used to exclude weak hyperfine components with brightness temperatures lower than the noise. The distributions of $\alpha$ and $\beta$ are shown in Fig. 13, the values of $\alpha$ range from 0.45 to $3.39, \beta$ lies between 0.45 and 1.95, and $\gamma$ between 0.57 and 1.85. The median of $\alpha$ is $1.27 \pm 0.03$ with a standard deviation of 0.45 , a one-sample t-test shows a significant deviation of the peak in the distribution of $\alpha$ from 1 with a $p$-value $<0.0001$. While the median of $\beta$ of $0.9 \pm 0.02$ with a standard deviation of 0.3 and the median of $\gamma$ of $1.06 \pm 0.02$ with a standard deviation of 0.37 are closer to 1 , a one-sample t-test yields that the peaks of $\beta$ and $\gamma$ differ 


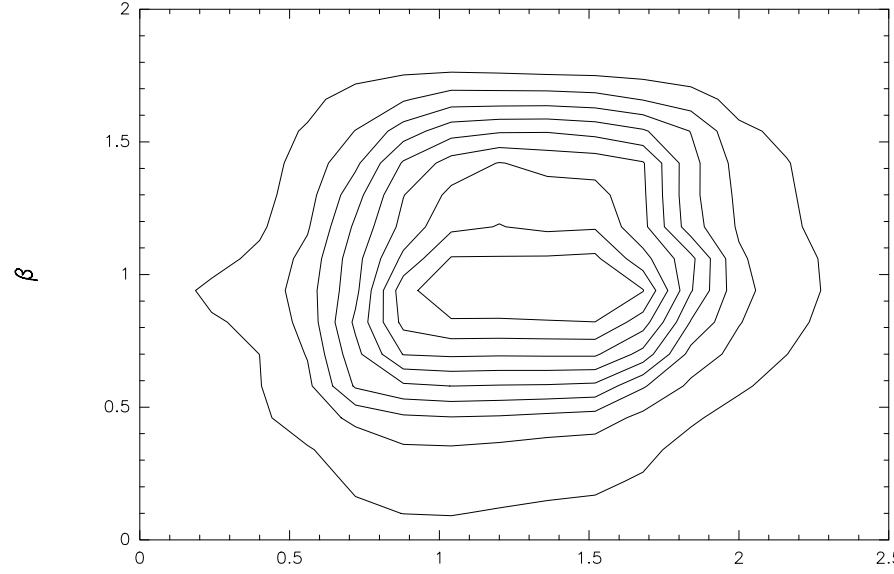

$\alpha$

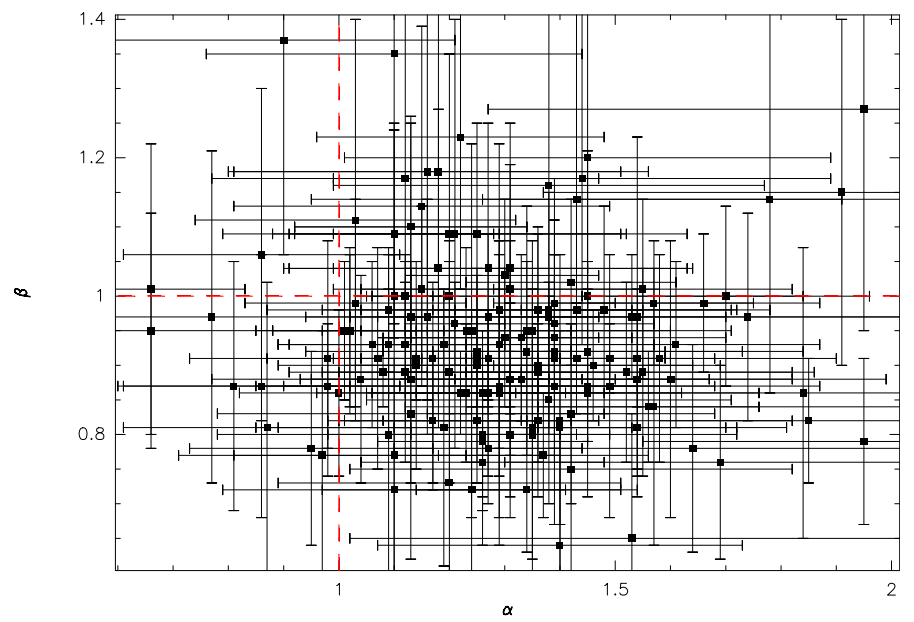

Fig. 14. Correlation plot of $\alpha$ and $\beta$. The contour plot in the upper panel shows no correlation between the two parameters. For this plot we counted the number of sources in each $\alpha$ and $\beta$ bin of 0.5. The scatter plot in the lower panel indicates that some $\alpha$ and $\beta$ values deviate from 1 , as shown by the dashed red lines.

significantly from 1 with a $p$-value $<0.0001$. Figure 13 also indicates that the distribution of $\alpha$ exhibits a much larger scatter than that of $\beta$ and $\gamma$, consistent with their standard deviations. Figure 14 presents our calculated estimates of $\beta$ plotted against our $\alpha$ values with straight lines indicating where the two parameters equal 1. Defining sources with hyperfine anomalies such as as those exhibiting $\alpha \pm \Delta \alpha$ or $\beta \pm \Delta \beta$ different from $1, \sim 61 \%$ of the observed $\mathrm{NH}_{3}(1,1)$ spectra in the fourth quadrant show hyperfine anomalies. We note that the hyperfine model of the $\mathrm{NH}_{3}(1,1)$ line taking all 18 hyperfine components into account leads to a splitting of the outer satellite lines into two components, separated by $0.5 \mathrm{~km} \mathrm{~s}^{-1}$ for $\mathrm{O} 1$ and by $0.14 \mathrm{~km} \mathrm{~s}^{-1}$ for O2. This can cause a difference of the temperature ratio $\alpha$ from 1 for narrow lines even without hyperfine anomalies. A hyperfine model including a small $\mathrm{NH}_{3}(1,1)$ line width of $1 \mathrm{~km} \mathrm{~s}^{-1}$, for example, leads to $\alpha$ of 1.18 , while the deviation from 1 is even smaller for an average $\mathrm{NH}_{3}(1,1)$ line width of $2 \mathrm{~km} \mathrm{~s}^{-1}$ in our sample, which leads to $\alpha$ of 1.03. Hence, this effect cannot account for the measured median of $\alpha, 1.27 \pm 0.03$ in our sample, which therefore indicates true hyperfine anomalies.

To assess the influence of the baseline subtraction on the significance of the hyperfine anomalies, we reduced the $\mathrm{NH}_{3}(1,1)$ spectra of the whole sample in the fourth quadrant again using different orders of the baseline. Two choices for an odd order of the baseline and also an even order lead to a variation of the hyperfine anomaly parameters by only 8 to $14 \%$, which demonstrates that the anomalies are real features.

Longmore et al. (2007) suggested that $\alpha>1, \beta=1$ for nonthermal excitation, while systematic motion are related to $\alpha, \beta<1$ for infall and $\alpha, \beta>1$ for outflow. The median values of $\alpha$ and $\beta$ and their comparison in Fig. 14 indicate that non-LTE conditions prevail in most clumps. This result is similar to that of Longmore et al. (2007), who analysed $\mathrm{NH}_{3}$ hyperfine anomalies in a much smaller sample of 21 southern hot molecular cores. Their comparison of $\alpha, \beta$, and $\gamma$ values in correlation plots indicates that the nonthermal excitation of the cores are more likely than systematic motion.

\section{Discussion}

\subsection{Comparison with nearby molecular clouds}

In this section we investigate how some ATLASGAL clumps compare with properties of nearby molecular clouds. To do this, we compared $\mathrm{NH}_{3}$ properties of ATLASGAL clumps with cores within a region in different nearby molecular clouds that corresponds to the Parkes beam width of $2 \mathrm{pc}$ at an average distance of the ATLASGAL sample of $5 \mathrm{kpc}$. Rosolowsky et al. (2008) observed $\mathrm{NH}_{3}(1,1)$ and $\mathrm{NH}_{3}(2,2)$ lines in the Perseus molecular cloud using the $100 \mathrm{~m}$ Robert F. Byrd Green Bank Telescope (GBT). Their beam width of $31^{\prime \prime}$ corresponds to 0.04 $\mathrm{pc}$ at a distance of $260 \mathrm{pc}$. Because we wish to compare the $\mathrm{NH}_{3}$ line parameters of the cores given in Rosolowsky et al. (2008) with those of our $\mathrm{NH}_{3}$ sample, we moved the cores to the same distance as the ATLASGAL sources. A region of 26' in the Perseus molecular cloud corresponds to the beam width of 2 pc used for the $\mathrm{NH}_{3}$ observations of ATLASGAL clumps. Calculating the mean $\mathrm{NH}_{3}$ properties of the cores within $26^{\prime}$, we obtain a velocity dispersion of $0.34 \mathrm{~km} \mathrm{~s}^{-1}$ for the subregion L1455/L1448, $0.41 \mathrm{~km} \mathrm{~s}^{-1}$ for NGC 1333, and $0.54 \mathrm{~km} \mathrm{~s}^{-1}$ for IC348. These values as well as the line widths of cores in the Perseus molecular cloud are lower than the mean $\mathrm{NH}_{3}(1,1)$ line width of ATLASGAL sources of $2 \mathrm{~km} \mathrm{~s}^{-1}$. Another still closer low-mass star-forming region is the Pipe Nebula at a distance of $130 \mathrm{pc}$. A survey of $\mathrm{NH}_{3}(1,1)$ and $(2,2)$ lines of starless cores in this molecular cloud complex was conducted by Rathborne et al. (2008) with the GBT. The beam width is $~ 30^{\prime \prime}$ and corresponds to $0.019 \mathrm{pc}$ at a distance of $130 \mathrm{pc}$. $\mathrm{NH}_{3}$ line parameters within $53^{\prime}$ are analysed to compare with our $\mathrm{NH}_{3}$ observations of ATLASGAL clumps. The velocity dispersion of the subregion located at $l \simeq 1^{\circ}$ and $b \simeq 4^{\circ}$ is $0.58 \mathrm{~km} \mathrm{~s}^{-1}$, while cores around $l \simeq-3^{\circ}$ and $b \simeq 7^{\circ}$ yield a lower velocity dispersion of $0.16 \mathrm{~km} \mathrm{~s}^{-1}$. These values are similar to those calculated in Perseus. In contrast to the two low-mass star-forming regions, the ATLASGAL sample has much larger line widths, indicating that different dynamics prevail in the high-mass starforming clumps, which are located in a more turbulent environment. In addition, $\mathrm{NH}_{3}(1,1)$ and $(2,2)$ lines were observed by Friesen et al. (2009) in the Ophiuchus molecular cloud, which is a nearby region of active clustered star formation. Friesen et al. (2009) combined their interferometer and single-dish telescope measurements using the Australia Telescope Compact Array, the Very Large Array, and the GBT. They thus obtained a beam width of $15^{\prime \prime}$, which corresponds to $0.009 \mathrm{pc}$ at a distance of $120 \mathrm{pc}$. A region of $56^{\prime}$ in Ophiuchus, which is comparable to the beam width of $2 \mathrm{pc}$ used for ATLASGAL sources, includes all observed cores that are located in the central Ophiuchus region. These exhibit a velocity dispersion of $0.32 \mathrm{~km} \mathrm{~s}^{-1}$ with line widths of up to $1 \mathrm{~km} \mathrm{~s}^{-1}$ that lie in the range of the smallest 
ATLASGAL line widths. Similar properties of cores in Ophiuchus and the ATLASGAL sample indicate that some ATLASGAL clumps could be low-mass cores. This also supports the process explaining $\mathrm{NH}_{3}$ hyperfine anomalies (see Sect. 4.3) by assuming that the observed line width of a molecular cloud results from the relative motion and small line widths of individual clumps.

The mean $\mathrm{NH}_{3}$ column density is similar in the three nearby molecular clouds and lower than that of the ATLASGAL sample. In Perseus we obtain a mean value of $5.3 \times 10^{14} \mathrm{~cm}^{-2}$ for L1455/L1448, $3.5 \times 10^{14} \mathrm{~cm}^{-2}$ for NGC 1333 and $1.6 \times$ $10^{14} \mathrm{~cm}^{-2}$ for IC348. The average ammonia column density in the Pipe Nebula is $\sim 3 \times 10^{14} \mathrm{~cm}^{-2}$, and Ophiuchus has a slightly higher mean value of $5.8 \times 10^{14} \mathrm{~cm}^{-2}$. It might be expected that the average column density measured within a larger region of the ATLASGAL clumps with a beam width of $40^{\prime \prime}$ is lower than the values obtained for the low-mass star-forming clouds with a smaller beam width of $\sim 30^{\prime \prime}$ and $15^{\prime \prime}$. However, the column density of the low-mass cores is still lower than that of the ATLASGAL sample with an average of $1.8 \times 10^{15} \mathrm{~cm}^{-2}$, which might result from a lower peak column density of the low-mass sources.

The mean kinetic temperature of the local molecular clouds is lower than that of ATLASGAL sources. We calculate mean values of $12.2 \mathrm{~K}$ for L1455/L1448 in Perseus, $14.3 \mathrm{~K}$ for NGC 1333 and $12.4 \mathrm{~K}$ for IC348. Cores in the Pipe Nebula have an average kinetic temperature of $\sim 12 \mathrm{~K}$ and Ophiuchus has a mean of $14 \mathrm{~K}$, while we obtain $20.8 \mathrm{~K}$ on average for the ATLASGAL sample. The mean kinetic temperature of ATLASGAL sources is calculated within a beam width of $2 \mathrm{pc}$, which is a more extended region around each clump compared to the smaller beam widths of the close molecular clouds. Hence, the lower kinetic temperatures of the low-mass cores are measured toward their peak, while the higher temperatures of ATLASGAL clumps indicate that they are in a warmer environment or that the more massive and luminous stars are better able to warm their surrounding. Moreover, the kinetic temperatures obtained for the ATLASGAL sample are similar to the temperatures measured for dust that is heated by the interstellar radiation field (Bernard et al. 2010).

\subsection{Virial parameters}

To determine virial masses, it is important that the line tracer used to calculate the velocity dispersion traces the same material as seen in the dust emission with ATLASGAL. Ideally, we would use maps to investigate whether there is a correlation between the extent of the regions probed by the molecular line and dust emission, but such data are not available for $\mathrm{NH}_{3}$. It is therefore useful to compare the velocity dispersion in different line tracers such as $\mathrm{N}_{2} \mathrm{H}^{+}$and $\mathrm{NH}_{3}$.

Gas masses and virial masses were compared for an ATLASGAL sample of 348 clumps observed in $\mathrm{NH}_{3}$, which have known distances from the GRS survey (Roman-Duval et al. 2009) or are located at the tangent points with similar near and far distances, in Wienen et al. (2012). This revealed smaller virial parameters with a mean of 0.21 for sources with narrow line widths, $\lesssim 1.8 \mathrm{~km} \mathrm{~s}^{-1}$, than for clumps exhibiting broad line widths, $\gtrsim 1.8 \mathrm{~km} \mathrm{~s}^{-1}$, with a mean virial parameter of 0.45 . We continue this study here with a comparison of virial parameters by using $\mathrm{NH}_{3}$ and the higher density tracer $\mathrm{N}_{2} \mathrm{H}^{+}$. We detect the $\mathrm{N}_{2} \mathrm{H}^{+}$line in 610 ATLASGAL sources, which is $87 \%$ of the observed southern sample. A subsample of 293 clumps in the fourth quadrant is detected in $\mathrm{N}_{2} \mathrm{H}^{+}$and $\mathrm{NH}_{3}$. Because the line widths of the two probes affect the virial mass estimates, we searched for trends of the line-width ratio. We corrected the line widths for the resolution of the spectrometer taking into account the channel width of $0.87 \mathrm{~km} \mathrm{~s}^{-1}$ of the Mopra telescope and of $0.4 \mathrm{~km} \mathrm{~s}^{-1}$ of the Parkes telescope with the correlation between the channels. This results in a $\mathrm{N}_{2} \mathrm{H}^{+}$ line width corrected for the velocity resolution, $\Delta v_{\mathrm{N}_{2} \mathrm{H}^{+}}$, corr $=$ $\sqrt{\Delta v_{\mathrm{N}_{2} \mathrm{H}^{+}}^{2}-\left(1.044 \mathrm{~km} \mathrm{~s}^{-1}\right)^{2}}$, and a deconvolved $\mathrm{NH}_{3}(1,1)$ line width, $\Delta v(1,1)_{\text {corr }}=\sqrt{\Delta v(1,1)^{2}-\left(0.4 \mathrm{~km} \mathrm{~s}^{-1}\right)^{2}}$. The lower panel of Fig. 15 shows the $\mathrm{N}_{2} \mathrm{H}^{+}$line widths corrected for the velocity resolution plotted against the deconvolved $\mathrm{NH}_{3}(1,1)$ line widths, the dotted straight line indicates equality. The solid

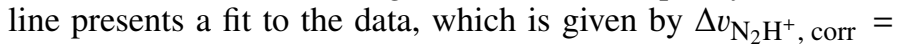
$0.81 \Delta v(1,1)_{\text {corr }}+1.26$. Both are intrinsic line widths from hyperfine structure fits. We took the seven hyperfine components into account for the fit of the $\mathrm{N}_{2} \mathrm{H}^{+}$lines. For the contour plot in the upper panel of Fig. 15 we chose a binning of $0.5 \mathrm{~km} \mathrm{~s}^{-1}$ for the deconvolved $\mathrm{NH}_{3}(1,1)$ and $\mathrm{N}_{2} \mathrm{H}^{+}$line widths. The $\mathrm{N}_{2} \mathrm{H}^{+}$ lines have widths between 0.9 and $7.7 \mathrm{~km} \mathrm{~s}^{-1}$ with a peak at $3 \mathrm{~km} \mathrm{~s}^{-1}$ and are therefore broader than the $\mathrm{NH}_{3}(1,1)$ lines with a peak at $2 \mathrm{~km} \mathrm{~s}^{-1}$. The difference in line width might indicate that the lines originate from different parts of the clumps. While $\mathrm{NH}_{3}$ probes densities of $\sim 10^{4} \mathrm{~cm}^{-3}$ (Ungerechts et al. 1986), the critical density of $\mathrm{N}_{2} \mathrm{H}^{+}$is $\sim 2 \times 10^{5} \mathrm{~cm}^{-3}$ (Aikawa et al. 2005). Assuming a density and temperature gradient over the extent of the sources, $\mathrm{N}_{2} \mathrm{H}^{+}$traces the denser inner region of a source and $\mathrm{NH}_{3}$ also probes the less dense envelope of the clump. In addition, the $\mathrm{N}_{2} \mathrm{H}^{+}$lines were measured with a smaller beam width of $38^{\prime \prime}$ compared to the $61^{\prime \prime}$ beam width of the $\mathrm{NH}_{3}$ observations. The narrower $\mathrm{NH}_{3}$ line widths measured with a larger beam width of the Parkes telescope compared to the $\mathrm{N}_{2} \mathrm{H}^{+}$line widths contradict the power-law relationship between the velocity dispersion and size of a cloud (Larson 1981). Such a trend was also found in a survey of massive clumps in the inner Galaxy by Wu et al. (2010a), who obtained larger line widths of the CS (7-6) transition than of the CS (2-1) line despite the much larger size of the CS (2-1) emission. However, the smaller inner part of a clump might be influenced by turbulence generated by heating or outflows, which causes broader line widths in the inner region probed by the higher density tracer. This is also presented in Fig. 16, which illustrates the $\mathrm{NH}_{3}$ rotational temperature plotted against the $\mathrm{NH}_{3}(1,1)$ to $\mathrm{N}_{2} \mathrm{H}^{+}$line-width ratio, taken as the mean of each bin of 0.15 . A low line-width ratio associated with low rotational temperatures is caused by a large $\mathrm{N}_{2} \mathrm{H}^{+}$line width probing the inner dense part of the source and a smaller width of the $\mathrm{NH}_{3}$ line, which is emitted over the whole extent of the clump. This region also includes the less dense and cold envelope, which likely results in a decrease in line width and rotational temperature by averaging over the source size. A linewidth ratio of $\sim 1$ and high rotational temperatures are associated with a higher density in the inner heated part of the clump, where turbulence is prevailing, $\mathrm{NH}_{3}$ and $\mathrm{N}_{2} \mathrm{H}^{+}$are excited and exhibit similar line widths.

To calculate the gas mass of a southern $\mathrm{NH}_{3}$ subsample of 280 sources with known distances, we used the same relation as for the northern clumps (see Eq. (12) in Wienen et al. 2012) with the $870 \mu \mathrm{m}$ flux density from the CSC, the kinematic distance to the complex, in which the source is located, given in Wienen et al. (2015), and the kinetic temperature listed in Table 3 . The virial masses are computed assuming virial equilibrium (Rohlfs \& Wilson 2004),

$M_{\mathrm{vir}}\left(M_{\odot}\right)=250 \Delta v(1,1)_{\mathrm{corr}}^{2} \times R$, 

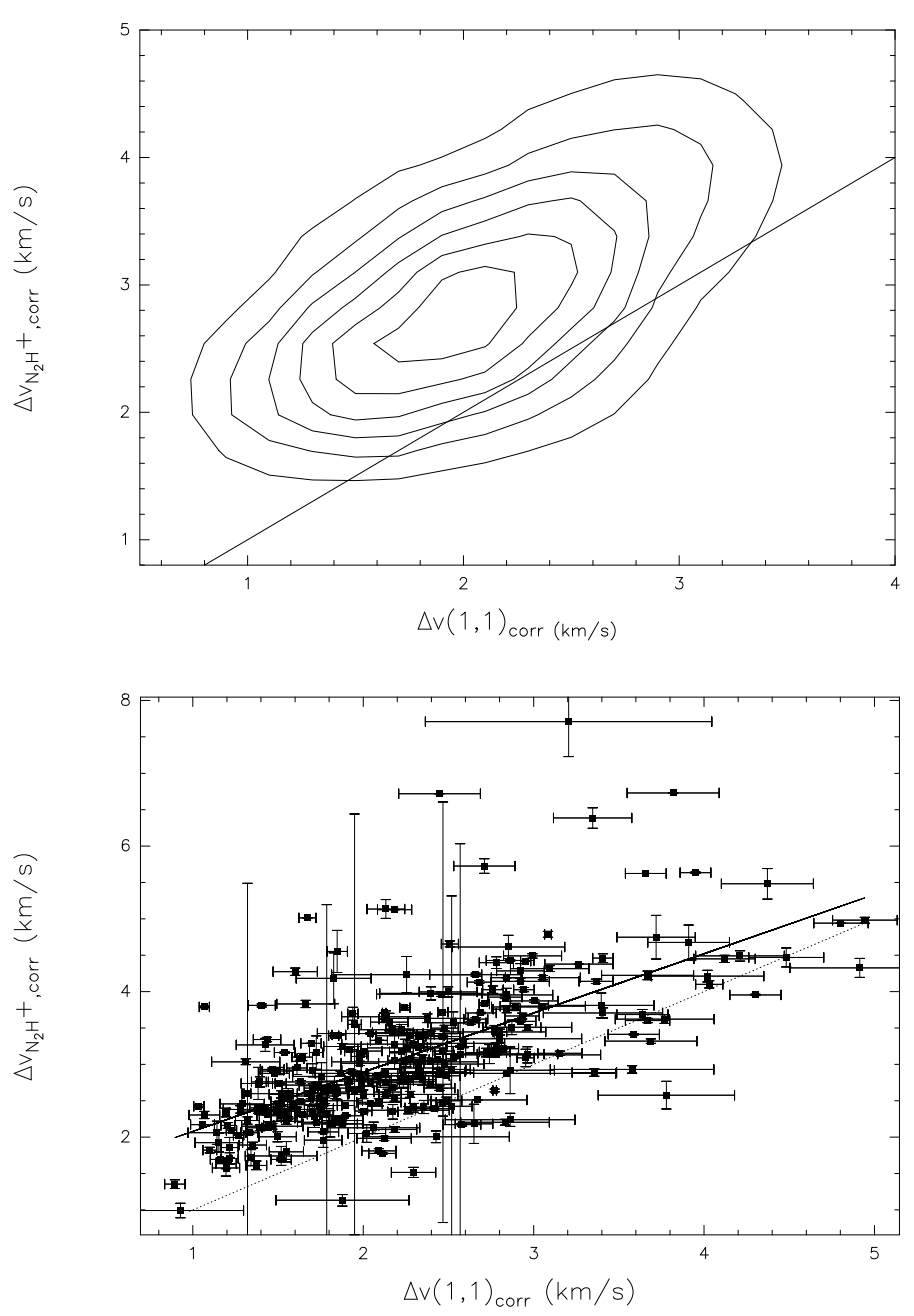

Fig. 15. Dependence of $\mathrm{N}_{2} \mathrm{H}^{+}$on $\mathrm{NH}_{3}$ line widths is shown as contour plot in the top panel and as a scatter plot in the lower panel. The binning of the two line widths in the contour plot is $0.5 \mathrm{~km} \mathrm{~s}^{-1}$. The dotted straight line indicates equality, the solid line denotes a fit to the data.

with the $\mathrm{NH}_{3}$ line width, $\Delta v(1,1)_{\text {corr }}$, in $\mathrm{km} \mathrm{s}^{-1}$ and the $\mathrm{N}_{2} \mathrm{H}^{+}$ line width, $\Delta v_{\mathrm{N}_{2} \mathrm{H}^{+}}$, corr , in $\mathrm{km} \mathrm{s}^{-1}$ corrected for the velocity resolution and the deconvolved effective radius in pc, as given in the CSC. We show the distribution of gas and virial masses calculated from the $\mathrm{NH}_{3}$ line width in black and from the $\mathrm{N}_{2} \mathrm{H}^{+}$ line width in red in Fig. 17. The black solid line indicates equality between gas and virial masses. The gas mass ranges from $\sim 10$ to $5 \times 10^{4} M_{\odot}$ with a peak at $\sim 2.5 \times 10^{3} M_{\odot}$, and the virial mass lies between $\sim 30$ and $3 \times 10^{4} M_{\odot}$ with a peak at $\sim 1000 M_{\odot}$ using the $\mathrm{NH}_{3}$ line width and a peak at $\sim 2000 M_{\odot}$ for values computed from the $\mathrm{N}_{2} \mathrm{H}^{+}$line width. We obtain on average higher gas masses than virial masses calculated from the $\mathrm{NH}_{3}$ line width, the same trend as found for the northern sample (Wienen et al. 2012). When we use the $\mathrm{N}_{2} \mathrm{H}^{+}$line width, the virial masses are higher than those resulting from the $\mathrm{NH}_{3}$ line width, and the red sample is well described by the black line. Higher virial masses also result in larger virial parameters, which are computed through the relation (Bertoldi \& McKee 1992)

$\alpha=\frac{M_{\mathrm{vir}}}{M_{\mathrm{gas}}}$.

We plot the logarithm of virial parameters against the logarithm of gas masses in Fig. 18. The lack of clumps with low gas masses and high virial parameters results from the limiting

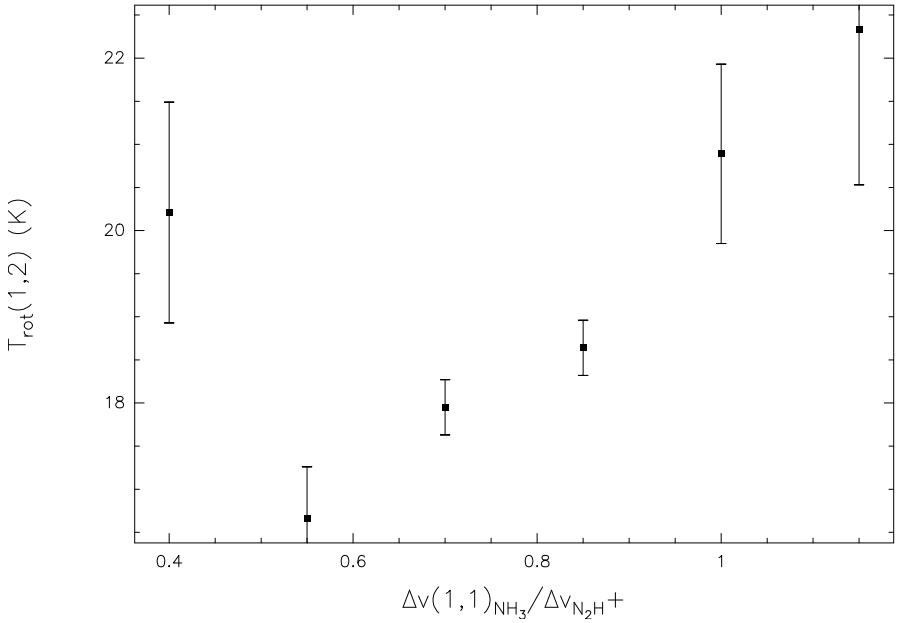

Fig. 16. Mean of the rotational temperature calculated in each bin of $\mathrm{NH}_{3}$ to $\mathrm{N}_{2} \mathrm{H}^{+}$line-width ratios of 0.15 and plotted against the line-width ratio.

sensitivity of the $\mathrm{NH}_{3}$ observations. The straight line illustrates $\alpha=1$ for sources, which are in hydrostatic equilibrium. Because the mean virial parameter is skewed to high values due to some sources that exhibit gas masses with large errors, we give the median $\alpha$ values. These are 0.54 for virial masses calculated using the $\mathrm{NH}_{3}$ line width, similar to the virial parameters of northern ATLASGAL sources observed in $\mathrm{NH}_{3}$ with a broad line width (Wienen et al. 2012) and 0.99 for estimates from the $\mathrm{N}_{2} \mathrm{H}^{+}$line width. We compare these values to the virial parameter of a sample of ATLASGAL sources observed in $\mathrm{C}^{17} \mathrm{O}$ (Giannetti et al. 2014). Although this molecule has a low critical density of $\sim 10^{3} \mathrm{~cm}^{-3}$ (Ungerechts et al. 1997), it also traces the dense part within a source because it is optically thin. The virial masses are determined using Eq. (10), the $\mathrm{C}^{17} \mathrm{O}$ line width corrected for the velocity resolution, $\Delta v_{\mathrm{C}^{17} \mathrm{O} \text {,corr }}=$ $\sqrt{\left(\Delta v_{\mathrm{C}^{17} \mathrm{O}}\right)^{2}-\left(0.64 \mathrm{~km} \mathrm{~s}^{-1}\right)^{2}}$, and the deconvolved effective radius from the CSC. The $\mathrm{C}^{17} \mathrm{O}$ line width leads to virial mass estimates that are similar to those using the $\mathrm{N}_{2} \mathrm{H}^{+}$line width, and to a median virial parameter of 0.8 . Previous studies have shown that the distribution in Fig. 18 can be fitted by a power law (Bertoldi \& McKee 1992; Lada et al. 2008). A fit to our data gives $\alpha \sim M^{-0.45 \pm 0.03}$ using $\mathrm{NH}_{3}, \alpha \sim M^{-0.41 \pm 0.03}$ for $\mathrm{N}_{2} \mathrm{H}^{+}$and $\alpha \sim M^{-0.49 \pm 0.13}$ for $\mathrm{C}^{17} \mathrm{O}$. The power-law slopes agree within the errors and are also consistent with the exponent of the $\mathrm{NH}_{3}$ sample in the first quadrant fitted by Kauffmann et al. (2013). Although the median virial parameters depend on the kind of tracer used, the slopes of the distributions are similar and in the narrow range between 0 and -1 as obtained for different star-forming samples by Kauffmann et al. (2013). ATLASGAL sources observed in $\mathrm{NH}_{3}$ are close to be virialised with a trend of a decreasing virial parameter with rising gas mass.

To investigate whether $\mathrm{NH}_{3}$ or $\mathrm{N}_{2} \mathrm{H}^{+}$better represents the mass of a clump determined through the dust properties, we compared the $\mathrm{H}_{2}$ volume density of ATLASGAL sources with the critical density of $\mathrm{NH}_{3}$ and $\mathrm{N}_{2} \mathrm{H}^{+}$. The $\mathrm{H}_{2}$ volume density of ATLASGAL sources with resolved kinematic distances (Wienen et al. 2015) lies between 500 and $2.8 \times 10^{5} \mathrm{~cm}^{-3}$ with a mean of $2 \times 10^{4} \mathrm{~cm}^{-3} . \mathrm{N}_{2} \mathrm{H}^{+}$has a critical density of $\sim 2 \times$ $10^{5} \mathrm{~cm}^{-3}$ (Aikawa et al. 2005). The study of the dust continuum at $1.2 \mathrm{~mm}$ and CS lines towards high-mass star-forming regions by Beuther et al. (2002) showed that the densities probed by high-density tracers are typically ten times higher than the 

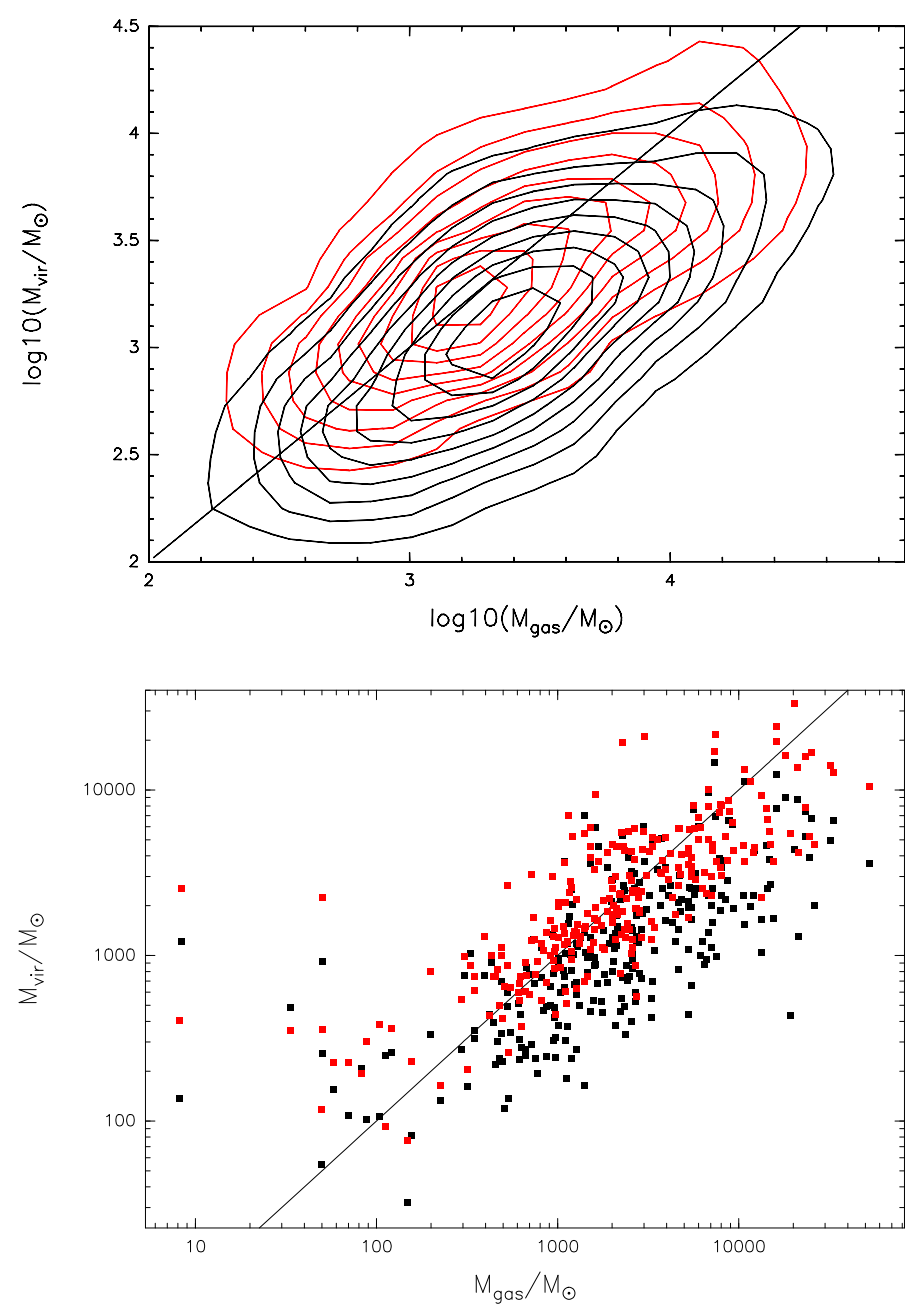

Fig. 17. Comparison of the logarithm of the virial mass and gas mass for masses derived from the $\mathrm{NH}_{3}$ line widths in black and from the $\mathrm{N}_{2} \mathrm{H}^{+}$ line widths in red. The contour plot of the two parameters is plotted in the upper panel with the range of the logarithm of the virial mass and gas mass divided into bins of 0.3 . The straight line shows equal masses.

densities measured from dust emission, which might result from fragmentation. $\mathrm{N}_{2} \mathrm{H}^{+}$could therefore trace several clumps of high densities within an ATLASGAL source. In contrast, the $\mathrm{NH}_{3}$ critical density is only $\sim 10^{4} \mathrm{~cm}^{-3}$ (Ungerechts et al. 1986) and it might probe a larger region than the dust emission. When we use the $\mathrm{NH}_{3}$ line width and the deconvolved effective radius derived from the $870 \mu \mathrm{m}$ dust continuum to estimate the virial mass, we find lower limits of the virial parameter because the radius is too small compared to the region of $\mathrm{NH}_{3}$ emission. However, $\mathrm{N}_{2} \mathrm{H}^{+}$might also trace a smaller region than the dust. To investigate whether there is a correlation between the two, we derived deconvolved effective radii from Gaussian fits to $\mathrm{N}_{2} \mathrm{H}^{+}$(1-0) maps observed by the MALT90, survey (The Millimetre Astronomy Legacy Team $90 \mathrm{GHz}$ Survey; Jackson et al. 2013), $\theta_{\mathrm{N}_{2} \mathrm{H}^{+}}$, and the deconvolved effective radii obtained from the $870 \mu \mathrm{m}$ dust emission given in the CSC, $\theta_{\text {submm }}$, for a sample of 105 ATLASGAL sources. Their comparison is displayed in Fig. 19, where the dotted straight line indicates equal effective radii. A fit to the data, shown by the solid line, yields $\theta_{\mathrm{N}_{2} \mathrm{H}^{+}}=1.22 \theta_{\text {submm }}+0.06$. While there is indication of larger $\mathrm{N}_{2} \mathrm{H}^{+}$radii than dust radii, they agree in $90 \%$ of the cases within $50 \%$. Statistically, $\mathrm{N}_{2} \mathrm{H}^{+}$therefore represents the size of the dust emission envelope well.
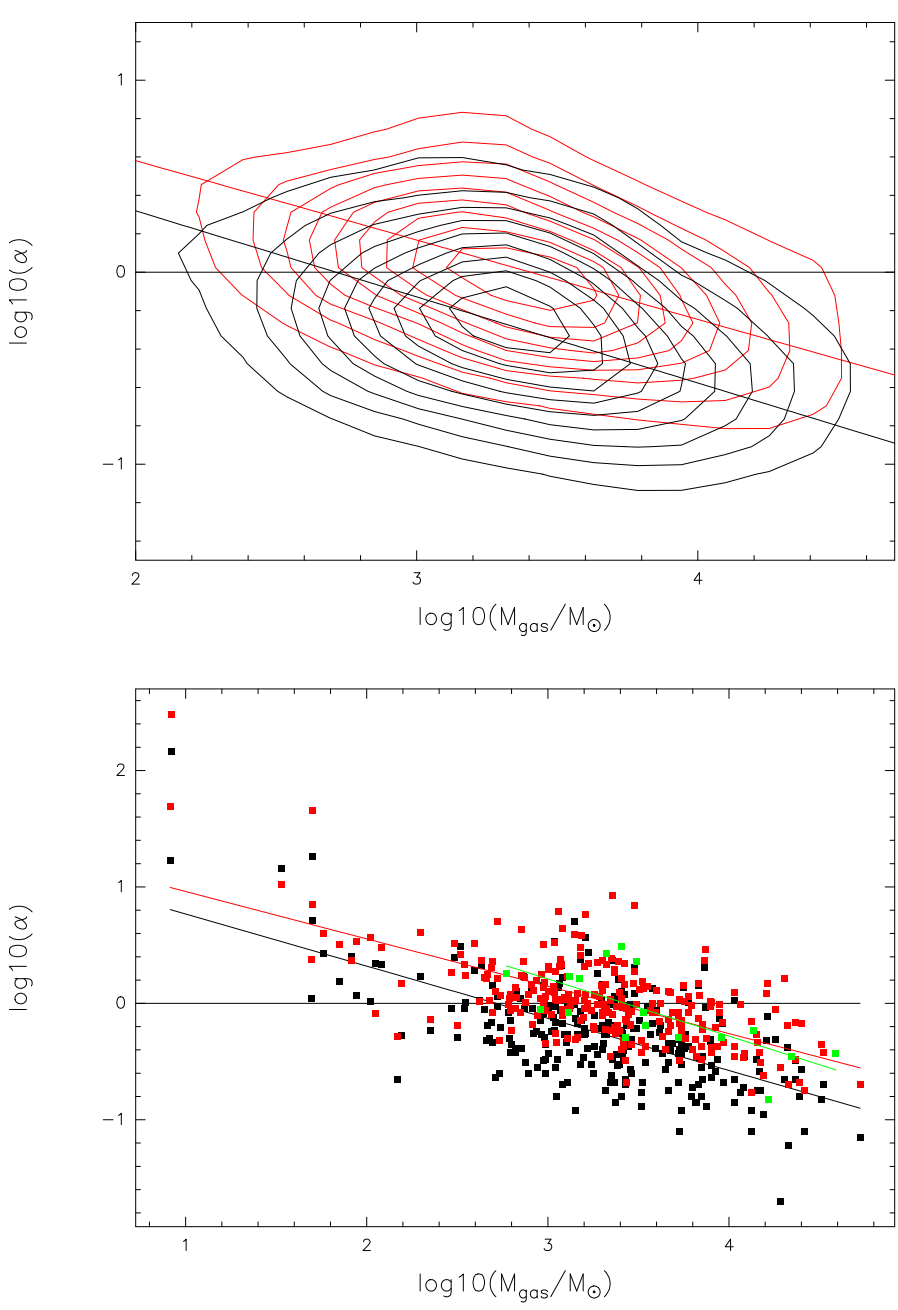

Fig. 18. Correlation plot of the logarithm of the virial parameter and the logarithm of the gas mass as black points for masses calculated using the $\mathrm{NH}_{3}$ line width, as red points using the $\mathrm{N}_{2} \mathrm{H}^{+}$line width, and as green points for the $\mathrm{C}^{17} \mathrm{O}$ line width. The horizontal black line indicates a virial parameter of 1 . A fit to each data set is shown as straight lines. The upper panel illustrates the contour plot, for which we counted the number of sources in each logarithmic gas mass and virial parameter bin of 0.3 in black for masses computed from the $\mathrm{NH}_{3}$ line width and in red for the $\mathrm{N}_{2} \mathrm{H}^{+}$line width.

In summary, our analysis of virial parameters obtained from different high-density tracers reveals that care is required in interpreting absolute values of the virial parameter. These estimates can also vary depending on the gas masses, which can change when different dust properties are used. There is a variation in turbulence within different parts of a source, which is related to different widths of spectral lines. $\mathrm{N}_{2} \mathrm{H}^{+}$exhibits broader line widths than $\mathrm{NH}_{3}$, leading to twice larger virial parameters. In addition, the size of the dust continuum and of the $\mathrm{N}_{2} \mathrm{H}^{+}$line emission agree well, which shows that the dust is better represented by $\mathrm{N}_{2} \mathrm{H}^{+}$than by $\mathrm{NH}_{3}$.

\subsection{Anomalies in the $\mathrm{NH}_{3}(1,1)$ quadrupole hyperfine structure}

It is expected that the inner and outer satellite intensity ratios of the $\mathrm{NH}_{3}(1,1)$ transition are symmetric in LTE. For optically thin hyperfine lines, the intensity ratio of the inner satellite line to the main line is expected to be 0.28 , while the ratio of the outer satellites to the main component is 0.22 (Ho \& Townes 1983). 


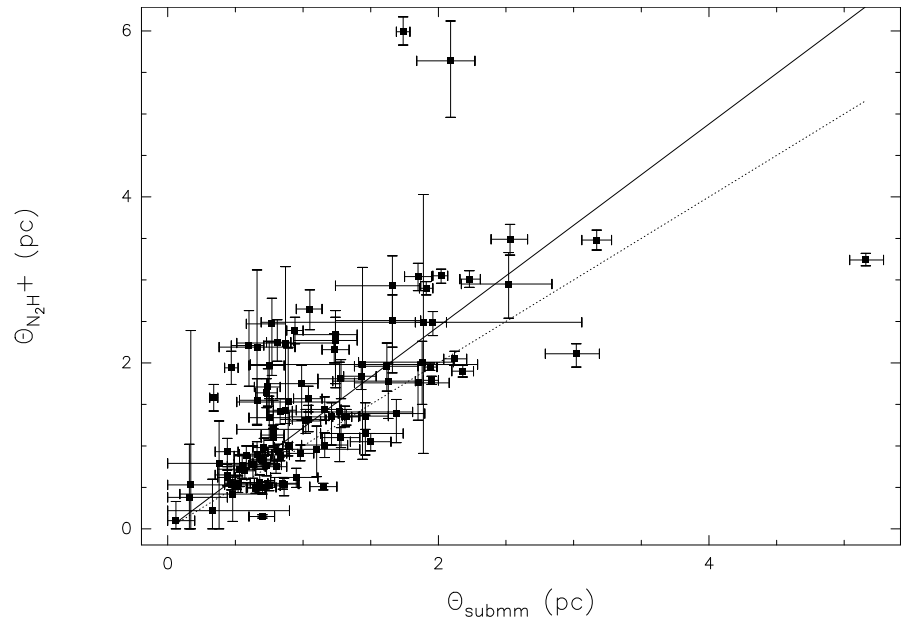

Fig. 19. Deconvolved effective radius derived from Gaussian fits to $\mathrm{N}_{2} \mathrm{H}^{+}$maps plotted against the radius determined from the $870 \mu \mathrm{m}$ emission. The dotted straight line denotes equal effective radii, the solid line shows a fit to the data.

However, $\sim 61 \%$ of the observed $\mathrm{NH}_{3}(1,1)$ spectra show deviations from this prediction. These hyperfine anomalies in the $\mathrm{NH}_{3}(1,1)$ emission have previously been observed towards several star-forming regions (Stutzki \& Winnewisser 1985; Matsakis et al. 1977; Stutzki et al. 1982).

One process that explains the nonthermal excitation of the $\mathrm{NH}_{3}(1,1)$ hyperfine levels is given by Matsakis et al. (1977). They assumed that the observed molecular cloud is clumped and the individual cores have small line widths $\left(\sim 0.3 \mathrm{~km} \mathrm{~s}^{-1}\right)$ and high densities $\left(10^{6}-10^{7} \mathrm{~cm}^{-3}\right)$. The relative motion of the clumps result then in the observed line width, and the measured line is an average over the clump spectra. The $\mathrm{NH}_{3}(1,1)$ hyperfine levels are populated mainly by the far IR $(J, K)=(2,1) \rightarrow$ $(1,1)$ photons, which are affected by selective trapping. The inner hyperfine satellites of the IR transition overlap with the main line, while the outer satellites are separated from those that are due to the small line widths. Consequently, it is more likely that photons in the outer satellite lines with a low optical depth can escape than those in the inner satellites with an increased optical depth. This leads to an overpopulation of the $\mathrm{NH}_{3}(1,1)$ outer hyperfine levels, which is stronger for the level with total angular momentum $F_{1}=0$ than for $F_{1}=1$ state. Because photons of the $F_{1}=0 \rightarrow 1$ transition come from a strongly overpopulated to a weaker populated level, the intensity of this outer satellite line on the red side (O2 in Fig. 12) is enhanced with regard to the intensity on the blue side $(\mathrm{O} 1), F_{1}=1 \rightarrow 0$, which goes to the strongly overpopulated $F_{1}=0$ state. The nonthermal excitation of the $(1,1)$ hyperfine levels explains anomalies in the outer satellite lines. However, some $\mathrm{NH}_{3}$ observations also show unequal intensities of the inner hyperfine structure lines, which are explained by Park (2001). They used Monte Carlo radiativetransfer calculations to investigate the influence of the velocity field from systematic motion such as expansion and contraction on the line anomaly. For a uniform core collapse with a velocity field $v(r) \propto r$, the photons emitted by an ammonia molecule and incident on another one are blueshifted. As a consequence, photons from a low-energy transition of the far IR $(\mathrm{J}, \mathrm{K})=(2,1) \longrightarrow$ $(1,1)$ decay can increase their energy and thus be reabsorbed, while higher energy photons leave the gas. The process works in the opposite way for systematic outflow. For different velocity fields such as inside-out collapse $\left(v(r) \propto r^{-0.5}\right)$, the procedure
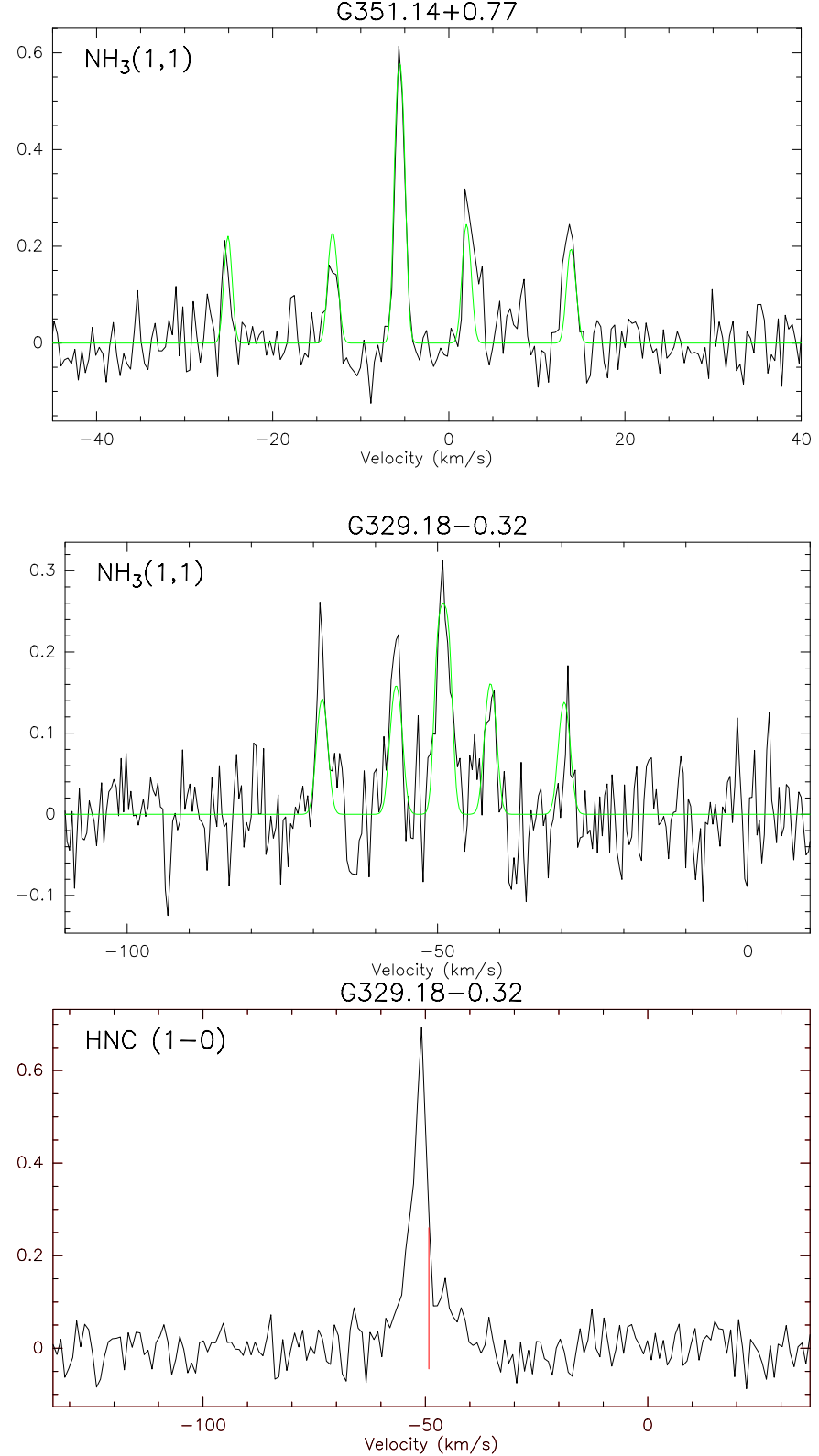

Fig. 20. $\mathrm{NH}_{3}(1,1)$ hyperfine anomaly of G351.14+0.77, which is illustrated in the upper panel, indicates a systematic outflow in this source. The middle panel displays the $\mathrm{NH}_{3}(1,1)$ line profile of G329.18-0.32, which indicates an infall. This is also supported by its HNC (1-0) line in the lowest panel with a stronger blue- than redshifted peak. The source velocity is shown by the straight red line.

is the same, although it is weaker because some photons are redshifted.

Section 3.8 describes our estimation of the hyperfine anomalies resulting in the three parameters $\alpha, \beta$, and $\gamma$ following the work by Longmore et al. (2007). The contour plot in Fig. 14 shows that there is no correlation between $\alpha$ and $\beta$, the scatter plot illustrates that $\alpha$ and $\beta$ of a few sources differ from 1 . Taking the errors of the parameters into account, the $\mathrm{NH}_{3}$ hyperfine anomaly might be produced by a systematic outflow in one source of our sample, G351.14+0.77, with $\alpha=1.65 \pm 0.63$ and $\beta=1.73 \pm 0.58$, while there is an indication of infall in one clump, G329.18-0.32, with $\alpha=0.45 \pm 0.26$ and $\beta=$ $0.71 \pm 0.27$, which results in the asymmetry of its satellite lines (see Fig. 20). Another indication of the infall in that source is the 
HNC (1-0) line profile shown in Fig. 20 in addition to the $\mathrm{NH}_{3}$ $(1,1)$ inversion transition. A blueshifted peak that is stronger than the redshifted peak is usually observed in an infalling envelope with a centrally peaked density and temperature distribution (Evans 1999). The blue peak originates from part of the cloud on the far side of the envelope, closer to the centre with a high excitation temperature, while the red peak is emitted from a point on the near side with a low excitation temperature. The central dip at the source LSR velocity is produced by self-absorption from low excited gas on the near side of the cloud.

We compared $\alpha$ and $\beta$ of the southern sources observed in $\mathrm{NH}_{3}$ with a massive star-forming subsample of clumps associated with an RMS source (Lumsden et al. 2013). However, no trend is found, which shows that on the scale of a clump, star formation processes such as outflow or infall driven by the embedded source do not influence the properties of the clumps.

Because our investigation yields that the hyperfine structure anomalies result from the nonthermal excitation of the $\mathrm{NH}_{3}$ $(1,1)$ levels populated by the far IR transition for most sources, we would expect a correlation between the asymmetry of the outer satellite lines and the luminosity of the source. We therefore searched for a correlation between $\alpha$ and the $\mathrm{NH}_{3}$ kinetic temperature as well as the ammonia $(1,1)$ line widths, which rise with increasing infrared luminosity of the source, but we did not find any correlation. We investigated whether the subsample with $\alpha, \beta<1$, which might indicate infall motion, exhibits lower virial masses than gas masses and has therefore the smallest virial parameters. We plot the logarithm of the virial parameter against the logarithm of the gas mass as presented in Fig. 18 for all ATLASGAL sources observed in $\mathrm{NH}_{3}$ in the fourth quadrant. A fit to the sources with $\alpha, \beta<1$ does not show whether the distribution is different from that of the whole sample because of the small number of clumps and the large uncertainties of the hyperfine component intensity ratios. Moreover, we analysed whether expanding sources associated with outflows are in a warmer and more turbulent environment. To do this, we searched for a trend of an increasing ratio $\alpha$ of the hyperfine components with rising line broadening $\Delta v(3,3) / \Delta v(1,1)$, but we found no correlation.

To summarize, our mean value of $\alpha$ of $1.29, \beta$ of 0.93 and $\gamma$ of 1.09 indicate that the nonthermal excitation of the $\mathrm{NH}_{3}(1,1)$ levels likely produces hyperfine structure anomalies. The parameters have large uncertainties and the comparison of $\alpha$ and $\beta$ shows no correlation between the two. The errors of $\alpha$ and $\beta$ we considered show that one source of our sample with $\alpha, \beta>1$ could be associated with an outflow and one clump with $\alpha, \beta<1$ with an infall.

\section{Summary}

We observed the $\mathrm{NH}_{3}(1,1)$ to $(3,3)$ inversion transitions of 354 dust clumps discovered by the ATLASGAL survey within a Galactic longitude from $300^{\circ}$ to $359^{\circ}$ and a latitude of $\pm 1.5^{\circ}$ using the Parkes telescope. Our main results can be summarized as follows:

1. We derived LSR velocities from the $\mathrm{NH}_{3}(1,1)$ lines mostly between 5 and $-120 \mathrm{~km} \mathrm{~s}^{-1}$. They are coincident with CO emission (Dame et al. 2001) and thus trace dense cores associated with large-scale molecular cloud structure. These velocities, together with those in the first quadrant (Wienen et al. 2012), are required to calculate near and far kinematic distances using the Brand \& Blitz (1993) rotation curve (Wienen et al. 2015).
2. The $\mathrm{NH}_{3}(1,1)$ line widths of the observed southern ATLASGAL sources, lying between 0.8 and $5 \mathrm{~km} \mathrm{~s}^{-1}$, are in a similar range as those of the northern clumps. We obtained mostly equal $(1,1)$ and $(2,2)$ line widths. For a subsample of sources with detected hyperfine satellites of the $(2,2)$ lines, we fitted the hyperfine structure using the $(2,2)$ optical depth derived from the $(1,1)$ optical depth and the $(2,2)$ to $(1,1)$ main-beam brightness temperature ratio. This led to broader $(2,2)$ line widths that those from a hyperfine structure fit with measured $(2,2)$ optical depth. Using the temperature ratio and the $(1,1)$ optical depth instead of the measured $(2,2)$ optical depth, we found an underestimation of the rotational temperature and column density by a factor 0.64 .

3. We analysed trends of $\mathrm{NH}_{3}$ line parameters vs galactocentric radius within the inner Galaxy. The rotational temperature, $\mathrm{H}_{2}$ column density and $\mathrm{NH}_{3}$ line widths show an approximately constant distribution. We obtained a decreasing $\mathrm{NH}_{3}$ abundance with galactocentric radius, consistent with the trend presented in Dunham et al. (2011). We investigated their assumption to explain this distribution also with a decreasing nitrogen abundance: the $\mathrm{N}_{2} \mathrm{H}^{+} / \mathrm{NH}_{3}$ column density ratio is constant within the inner Galaxy, in agreement with the modelling of a prestellar core (Hily-Blant et al. 2010), which indicates that an increase of the number of nitrogen atoms within a particular molecule might not lead to an enhanced decrease of its abundance.

4. $\mathrm{NH}_{3}$ line parameters of ATLASGAL clumps were compared with those of cores within nearby molecular clouds. We obtained smaller mean velocity dispersions of cores in low-mass star-forming regions such as the Perseus molecular cloud and the Pipe Nebula than the mean $\mathrm{NH}_{3}$ line width. This shows different dynamics between low- and high-mass star-forming clumps, while sources in the Ophiuchus molecular cloud associated with clustered star formation exhibit a velocity dispersion similar to the narrowest line widths of ATLASGAL sources. Lower mean values of the $\mathrm{NH}_{3}$ column density in nearby molecular clouds than the mean of the ATLASGAL sample might show a lower peak column density of low-mass cores. Moreover, the mean kinetic temperature within a smaller beam width towards nearby molecular clouds is lower than the average kinetic temperature in a larger beam width around each ATLASGAL clump. This indicates a warmer surrounding of ATLASGAL sources than that of low-mass cores.

5. Comparing the line widths of the $\mathrm{NH}_{3}(1,1)$ inversion transition, tracing densities of $\sim 10^{4} \mathrm{~cm}^{-3}$ (Ungerechts et al. 1986), with $\mathrm{N}_{2} \mathrm{H}^{+}$, probing higher densities of $\sim 2 \times 10^{5} \mathrm{~cm}^{-3}$ (Aikawa et al. 2005), shows broader $\mathrm{N}_{2} \mathrm{H}^{+}$than $\mathrm{NH}_{3}$ line widths. Moreover, the $\mathrm{NH}_{3} / \mathrm{N}_{2} \mathrm{H}^{+}$line-width ratio is increasing with rising rotational temperature. Broader $\mathrm{N}_{2} \mathrm{H}^{+}$than $\mathrm{NH}_{3}$ lines lead to higher virial masses with a median virial parameter of 1.03, compared to 0.54 resulting from the $\mathrm{NH}_{3}$ line width. Because the critical density and the radius derived from $\mathrm{N}_{2} \mathrm{H}^{+}$agree with the density and the radius of a dust clump determined from the $870 \mu \mathrm{m}$ emission, the dust is better represented by $\mathrm{N}_{2} \mathrm{H}^{+}$than by $\mathrm{NH}_{3}$.

6. We investigated deviations in the relative intensities of the $\mathrm{NH}_{3}(1,1)$ satellite lines from LTE to distinguish between the processes that result in these hyperfine structure anomalies. As described in Longmore et al. (2007), we calculated the ratio of the temperatures of the outer hyperfine components, $\alpha$, of the inner satellite lines, $\beta$, and of the sum of the outer and inner hyperfine structure lines on each side of the main line, $\gamma$. The median of $\alpha$ of $1.27 \pm 0.45$ differs significantly 
from 1 compared to a smaller deviation of the median values of $\beta$ of $0.9 \pm 0.3$ and of $\gamma$ of $1.06 \pm 0.37$ from 1 . These parameters show that the anomalies of most ATLASGAL clumps are likely explained by a nonthermal excitation of the $\mathrm{NH}_{3}(1,1)$ hyperfine levels. However, the intensity ratio of the satellite lines of two sources indicate systematic motion that results in the hyperfine structure anomalies.

7. In a second paper Wienen et al. (2015), we used the radial velocities derived in the first and fourth quadrant to resolve the kinematic distance ambiguity towards a large sample of high-mass star-forming regions discovered by ATLASGAL.

Acknowledgements. M.W. participates in the CSIRO Astronomy and Space Science Student Program and acknowledges support from the ATNF staff within the course of the observations. We thank the referee, Erik Rosolowsky, for a careful reading of this article and the very useful comments and suggestions.

\section{References}

Aguirre, J. E., Ginsburg, A. G., Dunham, M. K., et al. 2011, ApJS, 192, 4 Aikawa, Y., Herbst, E., Roberts, H., \& Caselli, P. 2005, ApJ, 620, 330 Aikawa, Y., Wakelam, V., Hersant, F., Garrod, R. T., \& Herbst, E. 2012, ApJ, 760,40

Anderson, L. D., Bania, T. M., Balser, D. S., \& Rood, R. T. 2011, ApJS, 194, 32 André, P., Ward-Thompson, D., \& Barsony, M. 2000, Protostars and Planets IV, 59

Bania, T. M. 1977, ApJ, 216, 381

Battisti, A. J., \& Heyer, M. H. 2014, ApJ, 780, 173

Becker, R. H., White, R. L., McLean, B. J., Helfand, D. J., \& Zoonematkermani, S. 1990, ApJ, 358, 485

Benjamin, R. A., Churchwell, E., Babler, B. L., et al. 2003, PASP, 115, 953

Benson, P. J., Caselli, P., \& Myers, P. C. 1998, ApJ, 506, 743

Bergin, E. A., \& Tafalla, M. 2007, ARA\&A, 45, 339

Bernard, J.-P., Paradis, D., Marshall, D. J., et al. 2010, A\&A, 518, L88

Bertin, E., \& Arnouts, S. 1996, A\&AS, 117, 393

Bertoldi, F., \& McKee, C. F. 1992, ApJ, 395, 140

Beuther, H., Schilke, P., Menten, K. M., et al. 2002, ApJ, 566, 945

Bontemps, S., Motte, F., Csengeri, T., \& Schneider, N. 2010, A\&A, 524, A18

Brand, J., \& Blitz, L. 1993, A\&A, 275, 67

Brand, J., Cesaroni, R., Palla, F., \& Molinari, S. 2001, A\&A, 370, 230

Brogan, C. L., Hunter, T. R., Cyganowski, C. J., et al. 2011, ApJ, 739, L16

Bronfman, L., Cohen, R. S., Alvarez, H., May, J., \& Thaddeus, P. 1988, ApJ, 324, 248

Bronfman, L., Nyman, L.-A., \& May, J. 1996, A\&AS, 115, 81

Burton, W. B., Gordon, M. A., Bania, T. M., \& Lockman, F. J. 1975, ApJ, 202 30

Carey, S. J., Clark, F. O., Egan, M. P., et al. 1998, ApJ, 508, 721

Carey, S. J., Feldman, P. A., Redman, R. O., et al. 2000, ApJ, 543, L157

Carey, S. J., Noriega-Crespo, A., Mizuno, D. R., et al. 2009, PASP, 121, 76

Caswell, J. L., Vaile, R. A., Ellingsen, S. P., Whiteoak, J. B., \& Norris, R. P. 1995, MNRAS, 272, 96

Caswell, J. L., Fuller, G. A., Green, J. A., et al. 2010, MNRAS, 404, 1029

Caswell, J. L., Fuller, G. A., Green, J. A., et al. 2011, MNRAS, 417, 1964

Cesaroni, R., Churchwell, E., Hofner, P., Walmsley, C. M., \& Kurtz, S. 1994, A\&A, 288, 903

Chira, R.-A., Beuther, H., Linz, H., et al. 2013, A\&A, 552, A40

Clark, J. S., \& Porter, J. M. 2004, A\&A, 427, 839

Cohen, R. S., \& Thaddeus, P. 1977, ApJ, 217, L155

Contreras, Y., Schuller, F., Urquhart, J. S., et al. 2013, A\&A, 549, A45

Csengeri, T., Bontemps, S., Schneider, N., et al. 2011, ApJ, 740, L5

Csengeri, T., Urquhart, J. S., Schuller, F., et al. 2014, A\&A, 565, A75

Dame, T. M., Hartmann, D., \& Thaddeus, P. 2001, ApJ, 547, 792

Duarte-Cabral, A., Bontemps, S., Motte, F., et al. 2013, A\&A, 558, A125

Duarte-Cabral, A., Bontemps, S., Motte, F., et al. 2014, A\&A, 570, A1

Dunham, M. K., Rosolowsky, E., Evans, II, N. J., et al. 2010, ApJ, 717, 1157

Dunham, M. K., Rosolowsky, E., Evans, II, N. J., Cyganowski, C., \& Urquhart, J. S. 2011, ApJ, 741, 110

Eden, D. J., Moore, T. J. T., Morgan, L. K., Thompson, M. A., \& Urquhart, J. S. 2013, MNRAS, 431, 1587

Egan, M. P., Shipman, R. F., Price, S. D., et al. 1998, ApJ, 494, L199

Evans, II, N. J. 1999, ARA\&A, 37, 311

Fontani, F., Cesaroni, R., Testi, L., et al. 2004, A\&A, 414, 299
Friesen, R. K., Di Francesco, J., Shirley, Y. L., \& Myers, P. C. 2009, ApJ, 697, 1457

Giannetti, A., Brand, J., Sánchez-Monge, Á., et al. 2013, A\&A, 556, A16

Giannetti, A., Wyrowski, F., Brand, J., et al. 2014, A\&A, 570, A65

Green, J. A., Caswell, J. L., Fuller, G. A., et al. 2009, MNRAS, 392, 783

Guilloteau, S., Wilson, T. L., Batrla, W., Martin, R. N., \& Pauls, T. A. 1983, A\&A, 124, 322

Hily-Blant, P., Walmsley, M., Pineau Des Forêts, G., \& Flower, D. 2010, A\&A, 513, A41

Hindson, L., Thompson, M. A., Urquhart, J. S., et al. 2013, MNRAS, 435, 2003

Ho, P. T. P., \& Townes, C. H. 1983, ARA\&A, 21, 239

Hoare, M. G., Purcell, C. R., Churchwell, E. B., et al. 2012, PASP, 124, 939

Hunter, T. R., Churchwell, E., Watson, C., et al. 2000, AJ, 119, 2711

Hunter, T. R., Brogan, C. L., Indebetouw, R., \& Cyganowski, C. J. 2008, ApJ, 680, 1271

Jackson, J. M., Rathborne, J. M., Foster, J. B., et al. 2013, PASA, 30, 57

Johnstone, D., Rosolowsky, E., Tafalla, M., \& Kirk, H. 2010, ApJ, 711, 655

Kauffmann, J., Pillai, T., \& Goldsmith, P. F. 2013, ApJ, 779, 185

Kraemer, K. E., \& Jackson, J. M. 1995, ApJ, 439, L9

Kramer, C., Stutzki, J., Rohrig, R., \& Corneliussen, U. 1998, A\&A, 329, 249

Kroupa, P., Weidner, C., Pflamm-Altenburg, J., et al. 2013, in Planets, Stars and Stellar Systems, eds. T. D. Oswalt, \& G. Gilmore (Dordrecht: Springer Science+Business Media), 5, 115

Kurtz, S., Churchwell, E., \& Wood, D. O. S. 1994, ApJS, 91, 659

Lada, C. J., Muench, A. A., Rathborne, J., Alves, J. F., \& Lombardi, M. 2008, ApJ, 672, 410

Ladd, N., Purcell, C., Wong, T., \& Robertson, S. 2005, PASA, 22, 62

Larson, R. B. 1981, MNRAS, 194, 809

Le Bourlot, J. 1991, A\&A, 242, 235

Lee, E. J., Murray, N., \& Rahman, M. 2012, ApJ, 752, 146

Longmore, S. N., Burton, M. G., Barnes, P. J., et al. 2007, MNRAS, 379, 535

Lu, X., Zhang, Q., Liu, H. B., Wang, J., \& Gu, Q. 2014, ApJ, 790, 84

Lumsden, S. L., Hoare, M. G., Urquhart, J. S., et al. 2013, ApJS, 208, 11

Mangum, J. G., \& Wootten, A. 1994, ApJ, 428, L33

Mangum, J. G., Wootten, A., \& Mundy, L. G. 1992, ApJ, 388, 467

Matsakis, D. N., Brandshaft, D., Chui, M. F., et al. 1977, ApJ, 214, L67

Minier, V., André, P., Bergman, P., et al. 2009, A\&A, 501, L1

Molinari, S., Swinyard, B., Bally, J., et al. 2010, PASP, 122, 314

Moscadelli, L., \& Goddi, C. 2014, A\&A, 566, A150

Nejad, L. A. M., Williams, D. A., \& Charnley, S. B. 1990, MNRAS, 246, 183

Park, Y.-S. 2001, A\&A, 376, 348

Perault, M., Omont, A., Simon, G., et al. 1996, A\&A, 315, L165

Peretto, N., \& Fuller, G. A. 2009, A\&A, 505, 405

Pestalozzi, M. R., Minier, V., \& Booth, R. S. 2005, A\&A, 432, 737

Pillai, T., Wyrowski, F., Carey, S. J., \& Menten, K. M. 2006, A\&A, 450, 569

Plume, R., Jaffe, D. T., Evans, II, N. J., Martín-Pintado, J., \& Gómez-González, J. 1997, ApJ, 476, 730

Purcell, C. R., Longmore, S. N., Walsh, A. J., et al. 2012, MNRAS, 426, 1972

Ragan, S. E., Bergin, E. A., \& Wilner, D. 2011, ApJ, 736, 163

Rathborne, J. M., Lada, C. J., Muench, A. A., Alves, J. F., \& Lombardi, M. 2008, ApJS, 174, 396

Rodriguez, L. F., Canto, J., \& Moran, J. M. 1982, ApJ, 255, 103

Rohlfs, K., \& Wilson, T. L. 2004, Tools of radio astronomy (Berlin; New York: Springer)

Rolleston, W. R. J., Smartt, S. J., Dufton, P. L., \& Ryans, R. S. I. 2000, A\&A, 363,537

Roman-Duval, J., Jackson, J. M., Heyer, M., et al. 2009, ApJ, 699, 1153

Rosolowsky, E. W., Pineda, J. E., Foster, J. B., et al. 2008, ApJS, 175, 509

Scalise, Jr., E., Rodriguez, L. F., \& Mendoza-Torres, E. 1989, A\&A, 221, 105

Schuller, F., Menten, K. M., Contreras, Y., et al. 2009, A\&A, 504, 415

Sevenster, M. N., Chapman, J. M., Habing, H. J., Killeen, N. E. B., \& Lindqvist, M. 1997, A\&AS, 124, 509

Shaver, P. A., McGee, R. X., Newton, L. M., Danks, A. C., \& Pottasch, S. R. 1983, MNRAS, 204, 53

Simon, R., Rathborne, J. M., Shah, R. Y., Jackson, J. M., \& Chambers, E. T. 2006, ApJ, 653, 1325

Siringo, G., Weiss, A., Kreysa, E., et al. 2007, The Messenger, 129, 2

Siringo, G., Kreysa, E., Kovacs, A., et al. 2008, in SPIE Conf. Ser., 7020, 3

Sridharan, T. K., Beuther, H., Schilke, P., Menten, K. M., \& Wyrowski, F. 2002, ApJ, 566, 931

Stutzki, J., \& Guesten, R. 1990, ApJ, 356, 513

Stutzki, J., \& Winnewisser, G. 1985, A\&A, 144, 13

Stutzki, J., Ungerechts, H., \& Winnewisser, G. 1982, A\&A, 111, 201

Szymczak, M., Kus, A. J., Hrynek, G., Kěpa, A., \& Pazderski, E. 2002, A\&A, 392, 277

Tafalla, M., Myers, P. C., Caselli, P., Walmsley, C. M., \& Comito, C. 2002, ApJ, 569,815

Ungerechts, H., Winnewisser, G., \& Walmsley, C. M. 1986, A\&A, 157, 207 
Ungerechts, H., Bergin, E. A., Goldsmith, P. F., et al. 1997, ApJ, 482, 245

Urquhart, J. S., Busfield, A. L., Hoare, M. G., et al. 2007, in IAU Symp. 237, eds. B. G. Elmegreen, \& J. Palous, 482

Urquhart, J. S., Morgan, L. K., Figura, C. C., et al. 2011, MNRAS, 418, 1689

Urquhart, J. S., Moore, T. J. T., Schuller, F., et al. 2013a, MNRAS, 431, 1752

Urquhart, J. S., Thompson, M. A., Moore, T. J. T., et al. 2013b, MNRAS, 435 , 400

Urquhart, J. S., Csengeri, T., Wyrowski, F., et al. 2014a, A\&A, 568, A41

Urquhart, J. S., Figura, C. C., Moore, T. J. T., et al. 2014b, MNRAS, 437, 1791

Urquhart, J. S., Figura, C. C., Moore, T. J. T., et al. 2015, MNRAS, 452, 4029

van der Tak, F. F. S., Black, J. H., Schöier, F. L., Jansen, D. J., \& van Dishoeck, E. F. 2007, A\&A, 468, 627

Vasyunina, T., Linz, H., Henning, T., et al. 2009, A\&A, 499, 149

Walmsley, C. M., \& Ungerechts, H. 1983, A\&A, 122, 164
Walsh, A. J., Burton, M. G., Hyland, A. R., \& Robinson, G. 1998, MNRAS, 301, 640

Walsh, A. J., Breen, S. L., Britton, T., et al. 2011, MNRAS, 416, 1764

Wienen, M., Wyrowski, F., Schuller, F., et al. 2012, A\&A, 544, A146

Wienen, M., Wyrowski, F., Menten, K. M., et al. 2015, A\&A, 579, A91

Wilson, T. L., Gaume, R. A., \& Johnston, K. J. 1993, ApJ, 402, 230

Womack, M., Ziurys, L. M., \& Wyckoff, S. 1992, ApJ, 387, 417

Wood, D. O. S., \& Churchwell, E. 1989, ApJS, 69, 831

Wu, J., Evans, II, N. J., Shirley, Y. L., \& Knez, C. 2010a, ApJS, 188, 313

Wu, Y. W., Xu, Y., Pandian, J. D., et al. 2010b, ApJ, 720, 392

Zhang, C.-P., Wang, J.-J., Xu, J.-L., Wyrowski, F., \& Menten, K. M. 2014, ApJ, 784, 107

Zhang, Q., Hunter, T. R., Sridharan, T. K., \& Cesaroni, R. 1999, ApJ, 527, L117 